\title{
TITLE:
}

\section{Geometric approach to Lyapunov analysis in Hamiltonian dynamics}

$\operatorname{AUTHOR}(S)$ :

Yamaguchi, Yoshiyuki Y.; Iwai, Toshihiro

\section{CITATION:}

Yamaguchi, Yoshiyuki Y....[et al]. Geometric approach to Lyapunov analysis in Hamiltonian dynamics. Physical Review E 2001, 64(6): 066206.

\section{ISSUE DATE:}

2001-11-27

URL:

http://hdl.handle.net/2433/53452

RIGHT:

Copyright 2001 American Physical Society 
PHYSICAL REVIEW E, VOLUME 64, 066206

\title{
Geometric approach to Lyapunov analysis in Hamiltonian dynamics
}

\author{
Yoshiyuki Y. Yamaguchi* and Toshihiro Iwai ${ }^{\dagger}$ \\ Department of Applied Mathematics and Physics, Kyoto University, Kyoto, 606-8501, Japan \\ (Received 3 April 2001; revised manuscript received 6 August 2001; published 27 November 2001)
}

\begin{abstract}
As is widely recognized in Lyapunov analysis, linearized Hamilton's equations of motion have two marginal directions for which the Lyapunov exponents vanish. Those directions are the tangent one to a Hamiltonian flow and the gradient one of the Hamiltonian function. To separate out these two directions and to apply Lyapunov analysis effectively in directions for which Lyapunov exponents are not trivial, a geometric method is proposed for natural Hamiltonian systems, in particular. In this geometric method, Hamiltonian flows of a natural Hamiltonian system are regarded as geodesic flows on the cotangent bundle of a Riemannian manifold with a suitable metric. Stability/instability of the geodesic flows is then analyzed by linearized equations of motion that are related to the Jacobi equations on the Riemannian manifold. On some geometric settings on the cotangent bundle, it is shown that along a geodesic flow in question, there exist Lyapunov vectors such that two of them are in the two marginal directions and the others orthogonal to the marginal directions. It is also pointed out that Lyapunov vectors with such properties cannot be obtained in general by the usual method that uses linearized Hamilton's equations of motion. Furthermore, it is observed from numerical calculation for a model system that Lyapunov exponents calculated in both methods, geometric and usual, coincide with each other, independently of the choice of the methods.
\end{abstract}

DOI: 10.1103/PhysRevE.64.066206

PACS number(s): 05.45.Jn, 02.40.Ky, 02.90.+p

\section{INTRODUCTION}

Natural Hamiltonian systems with many degrees of freedom have Hamiltonian functions of the form

$$
H(q, p)=\frac{1}{2} \sum_{i, j}^{N} \delta^{i j} p_{i} p_{j}+V(q) .
$$

In spite of the simple appearance, those Hamiltonian functions having appropriately chosen potential functions are used in a wide variety of physical sciences such as plasma physics, condensed matter physics, and celestial mechanics. However, the potential functions describe nonlinear interactions, in general, so that chaotic or highly unstable trajectories take place in respective phase spaces, as is widely recognized. The exponential instability of trajectories are measured in terms of Lyapunov exponents, which describe time-averaged properties of chaotic trajectories. Further, in the study of directional deviations of chaotic trajectories, Lyapunov vectors will be of great use.

The Lyapunov exponents and the Lyapunov vectors are defined through linearized Hamilton's equations of motion. For the Hamiltonian (1), the linearized equations take the form

$$
\frac{\mathrm{d} Q^{i}}{\mathrm{~d} t}=P_{i}, \quad \frac{\mathrm{d} P_{i}}{\mathrm{~d} t}=-\sum_{j=1}^{N} \frac{\partial^{2} V}{\partial q^{i} \partial q^{j}}[q(t)] Q^{j}, \quad i=1, \ldots, N,
$$

where $\mathbf{X}=\left(Q^{1}, \ldots, Q^{N}, P_{1}, \ldots, P_{N}\right)$ is a $2 N$-dimensional vector representing a deviation from a reference trajectory $(q(t), p(t))$ to a nearby trajectory. The linearized Eqs. (2) have $2 N$ linearly independent solutions, which we denote by

\footnotetext{
*Email address: yyama@amp.i.kyoto-u.ac.jp

†Email address: iwai@amp.i.kyoto-u.ac.jp
}

$\mathbf{X}_{a}(t), a=1, \ldots, 2 N$. The Lyapunov vectors $\mathbf{V}_{a}(t)$ are then obtained by orthogonalizing these solutions on the GramSchmidt method

$$
\mathbf{V}_{a}(t)=\mathbf{X}_{a}(t)-\sum_{b=1}^{a-1} \frac{\left\langle\mathbf{X}_{a}(t), \mathbf{V}_{b}(t)\right\rangle}{\left\langle\mathbf{V}_{b}(t), \mathbf{V}_{b}(t)\right\rangle} \mathbf{V}_{b}(t), \quad a=1, \ldots, 2 N
$$

where $\langle\mathbf{X}, \mathbf{V}\rangle$ denotes the inner product of $\mathbf{X}$ and $\mathbf{V}$. The $a$ th Lyapunov exponent $\lambda_{a}$ is calculated as

$$
\lambda_{a}=\lim _{t \rightarrow \infty} \frac{1}{t} \ln \frac{\left\|\mathbf{V}_{a}(t)\right\|}{\left\|\mathbf{V}_{a}(0)\right\|}
$$

It is to be noted that the values of the Lyapunov exponents are known to be independent of the choice of initial values of the Lyapunov vectors except for vanishing Lebesgue measure [1,2], and that the exponents are ordered as $\lambda_{1} \geqslant \lambda_{2}$ $\geqslant \cdots \geqslant \lambda_{2 N}$.

Since the Lyapunov exponents are time-averaged quantities, they are suitable for the study of statistic properties of Hamiltonian systems. For example, phase transitions are investigated by the use of Lyapunov exponents. In fact, the second-order phase transition [3] and the Kosterlitz-Thouless transition [4] are characterized by the discontinuity in the largest Lyapunov exponents and by a sudden change in the gradient of the largest Lyapunov exponent against energy, respectively. Further, the sum of all positive Lyapunov exponents, which is also viewed as a function of energy, is used in the discussion of a dynamical phase transition [5], according to which trajectory's phase transition from nearly integrable behavior to chaotic behavior occurs in an energy region in which the sum of positive exponents breaks into a rapid increase against energy. In contrast with this, the 
Lyapunov vectors are expected to be useful in studying dynamical behavior of chaotic trajectories, since they serve as time series [6,7].

Suppose that a reference trajectory is given in a phase space. Then, according to Eq. (3), one can form $2 N$ linearly independent Lyapunov vectors from solutions to the linearized equations of motion along the reference trajectory. However, two of the Lyapunov vectors that are associated with Lyapunov exponents $\lambda_{N}$ and $\lambda_{N+1}$ are considered as marginal, since $\lambda_{N}$ and $\lambda_{N+1}$ should vanish, as is widely recognized. One of those two Lyapunov vectors is the tangent vector to the trajectory $\mathbf{X}_{H}$ and the other the gradient vector of Hamiltonian function, $\operatorname{grad} H$. We may interpret these vectors as follows: The displacement in the direction $\mathbf{X}_{H}$ is regarded just as a certain time displacement in the reference trajectory, and the displacement in the direction $\operatorname{grad} H$ will give rise to a transfer to a nearby trajectory with an energy value different from that of the reference trajectory. In view of this, in order to analyze the instability of trajectories, we are allowed to require that the two directions pointed by the vectors $\mathbf{X}_{H}$ and $\operatorname{grad} H$ be separated out from the other $2 N-2$ directions. Put another way, the requirement means that a Lyapunov vector that is orthogonal to the plane spanned by $\mathbf{X}_{H}$ and $\operatorname{grad} H$ at an initial instant has to be orthogonal to the plane spanned by $\mathbf{X}_{H}$ and $\operatorname{grad} H$ at every instant. If the requirement is fulfilled, we will be able to discuss the instability of trajectories without influence of the two marginal directions.

Unfortunately, the usual method of Lyapunov analysis on the basis of Eqs. (2) does not satisfy the requirement in general. This is because for any solution $\mathbf{X}(t)$ to Eq. (2) one has

$$
\frac{\mathrm{d}}{\mathrm{d} t}\langle\mathbf{X}, \operatorname{grad} H\rangle=0
$$

so that one obtains $\langle\mathbf{X}, \operatorname{grad} H\rangle=0$ at any instant if $\left.\langle\mathbf{X}, \operatorname{grad} H\rangle\right|_{t=0}=0$ at an initial instant, but, in general, by no means can one make $\left\langle\mathbf{X}, \mathbf{X}_{H}\right\rangle$ vanish at any instant, so that even the first Lyapunov vector $\mathbf{V}_{1}$ cannot be made orthogonal to the plane spanned by $\mathbf{X}_{H}$ and $\operatorname{grad} H$ at every instant.

A way to construct Lyapunov vectors that satisfy the above-stated requirement is to adopt linearized equations of a different type from the usual one (2). To take a geometric approach to Hamilton's equations of motion is a step toward finding such Lyapunov vectors. As for the geometric approach, it is known that if the total energy of the natural dynamical system is fixed at $E$, Newton's equations of motion may be equivalently expressed as geodesic equations on a Riemannian manifold $\left(M, g_{i j}\right)$, where $M$ is a subspace of the configuration space $\mathbf{R}^{N}$ defined by $M=\left\{q \in \mathbf{R}^{N} \mid E\right.$ $-V(q)>0\}$ and $g_{i j}$ is the Jacobi metric defined by $g_{i j}(q)$ $=2[E-V(q)] \delta_{i j}$. Then the linearized equations of the geodesic equations are given by the Jacobi equations of the form

$$
\frac{\mathrm{d}^{2} X^{i}}{\mathrm{~d} s^{2}}+\sum_{j, k, l=1}^{N} R_{j k l}{ }^{i} X^{j} \frac{\mathrm{d} q^{k}}{\mathrm{~d} s} \frac{\mathrm{d} q^{l}}{\mathrm{~d} s}=0, \quad i=1, \ldots, N,
$$

where $R_{j k l}{ }^{i}$ are the components of the Riemann curvature tensor, and $s$ is the arc length defined as

$$
\mathrm{d} s^{2}=\sum_{i, j=1}^{N} g_{i j}(q) \mathrm{d} q^{i} \mathrm{~d} q^{j}=\sum_{i, j=1}^{N} g_{i j}(q) \frac{\mathrm{d} q^{i}}{\mathrm{~d} t} \frac{\mathrm{d} q^{j}}{\mathrm{~d} t} \mathrm{~d} t^{2} .
$$

On the other hand, the linearized Newton's equations are put in the form

$$
\frac{\mathrm{d}^{2} Q^{i}}{\mathrm{~d} t^{2}}+\sum_{j=1}^{N} \frac{\partial^{2} V}{\partial q^{i} \partial q^{j}} Q^{j}=0, \quad i=1, \ldots, N .
$$

Equations (6) and (8) are not transformed to each other through the parameter transformation (7), while Newton's equations of motion and geodesic equations for the Jacobi metric are transformed to each other. This geometric method has been introduced in the estimation of the largest Lyapunov exponent $\lambda_{1}$ with the aid of statistical mechanics [8-11]. They studied instability of geodesics through the Jacobi equation, a second-order differential equation, while the Lyapunov analysis needs first-order differential equations.

The geometric approach we will take in this paper is to be made on the cotangent bundle $T^{*} M$ of the Riemannian manifold $M$ in order to find first-order differential equations associated with Eq. (6) and thereby to construct Lyapunov vectors that satisfy the above-stated requirements. We will first work with generic linearized Hamilton's equations of motion on $T^{*} M$, and then specialize the resultant equations to linearized Hamilton's equations for geodesic flows on $T^{*} M$, which will be found to project to the Jacobi equations on $M$. Further, we will introduce a lifted metric on the cotangent bundle $T^{*} M$ to make it possible to discuss the orthogonality of vector fields on $T^{*} M$. The lifted metric may be called the Sasaki metric. On this setting, we will be able to find Lyapunov vectors satisfying the above-stated requirements along any geodesic flow on $T^{*} M$. Put in detail, it will be shown that along any geodesic flow on $T^{*} M$, there exist Lyapunov vectors such that those associated with the vanishing Lyapunov exponents $\lambda_{N}$ and $\lambda_{N+1}$ are $\mathbf{X}_{H}$ and $\operatorname{grad} H$, respectively, and the other $2 N-2$ Lyapunov vectors are all orthogonal to the plane spanned by $\mathbf{X}_{H}$ and $\operatorname{grad} H$ at each point of the geodesic flow.

This article is organized as follows: Section II contains a brief review of geodesics and Jacobi fields and, in particular, of the Jacobi metric, whose geodesics are equivalent to trajectories of the natural dynamical system with a fixed total energy. In Sec. II and succeeding sections, Einstein's summation convention is adopted, and we choose to denote by $\left(x^{i}\right)$ local coordinates on a general $m$-dimensional Riemannian manifold, and by $\left(q^{i}\right)$ the Cartesian coordinates on $\mathbf{R}^{N}$. Section III is concerned with geodesic flows on the cotangent bundle $T^{*} M$, which project to geodesics on $M$. To describe geodesic flows in a more geometric way, we introduce an adapted frame and a lifted Riemannian metric on $T^{*} M$. Linearized Hamilton's equations of motion are discussed in Sec. IV, and it will be shown that there exist Lyapunov vectors that satisfy the above-stated requirement along a geodesic flow on $T^{*} M$. Section $\mathrm{V}$ is for numerical calculations for a model system with three degrees of freedom. Lyapunov vectors and Lyapunov exponents are calculated numerically for the model system in both geometric and usual methods to 
compare the respective results. It will be shown that Lyapunov exponents calculated in respective methods coincide with each other, independently of the choice of methods. Section VI is devoted to concluding remarks. Appendices are attached in which related topics on geometry of cotangent bundles and a symplectic implicit Runge-Kutta method for numerical integration are reviewed. In particular, lifting vector fields on $M$ to $T^{*} M$ and the Levi-Civita connection with respect to the lifted metric on $T^{*} M$ are discussed.

\section{GEODESICS AND JACOBI FIELDS}

\section{A. Jacobi equations}

Let $(M, \mathbf{g})$ be an $m$-dimensional Riemannian manifold with metric $\mathbf{g}$. The metric induces the Levi-Civita connection $\nabla$ on $M$; for vector fields $\mathbf{Y}, \mathbf{Z} \in \bar{X}(M), \bar{X}(M)$ denoting the set of vector fields on $M$, the covariant derivative $\nabla_{\mathbf{Y}} \mathbf{Z}$ is defined, in terms of local coordinates $\left(x^{1}, \ldots, x^{m}\right)$, to be

$$
\nabla_{\mathbf{Y}} \mathbf{Z}=Y^{j}\left[\frac{\partial Z^{i}}{\partial x^{j}}+\Gamma_{j k}^{i} Z^{k}\right] \frac{\partial}{\partial x^{i}},
$$

where $\left(Y^{i}\right)$ and $\left(Z^{i}\right)$ are components of $\mathbf{Y}$ and $\mathbf{Z}$, respectively, the Christoffel symbols $\Gamma_{j k}^{i}$ are defined as

$$
\Gamma_{j k}^{i}=\frac{1}{2} g^{i \ell}\left(\frac{\partial g_{\ell k}}{\partial x^{j}}+\frac{\partial g_{j \ell}}{\partial x^{k}}-\frac{\partial g_{j k}}{\partial x^{\ell}}\right)
$$

with components of the metric

$$
g_{i j}=\mathbf{g}\left(\frac{\partial}{\partial x^{i}}, \frac{\partial}{\partial x^{j}}\right), \quad g_{i j} g^{j k}=\delta_{i}^{k} .
$$

For a geodesic $c(s)$ with $s$ the arc length parameter, the tangent vector $\boldsymbol{\xi}$ to the geodesic satisfies the geodesic equation

$$
\nabla_{\boldsymbol{\xi}} \boldsymbol{\xi}=\left.\left[\frac{\mathrm{d} \xi^{i}}{\mathrm{~d} s}+\Gamma_{j k}^{i} \xi^{j} \xi^{k}\right] \frac{\partial}{\partial x^{i}}\right|_{c(s)}=0
$$

where

$$
\boldsymbol{\xi}=\left.\frac{\mathrm{d} x^{i}}{\mathrm{~d} s} \frac{\partial}{\partial x^{i}}\right|_{c(s)}
$$

with

$$
\mathrm{d} s^{2}=g_{i j} \mathrm{~d} x^{i} \mathrm{~d} x^{j} .
$$

We are interested in stability/instability of geodesics. To this end, we consider a congruence of geodesics that looks like a fluid whose flow lines are geodesics with the $c(s)$ as a member of them. Then we may consider that the tangent vector $\boldsymbol{\xi}$ to $c(s)$ is extended to be a vector field defined in a neighborhood of the original geodesic $c(s)$. We may also assume that there exists a vector field $\mathbf{Y}$ satisfying the condition

$$
[\xi, \mathbf{Y}]=0
$$

in the same domain as that for $\boldsymbol{\xi}$. The condition (11) means that a geodesic with $\boldsymbol{\xi}$ its tangent vector is carried congruently to another infinitesimally nearby geodesic by the infinitesimal transformation $\mathbf{Y}$. Thus, $\mathbf{Y}$ is viewed as a deviation of geodesics. The vector field $\boldsymbol{\xi}$ may have singularity at which $\boldsymbol{\xi}$ is not defined uniquely, and $\mathbf{Y}$ may vanish there. With this in mind, we operate $\nabla_{\xi} \boldsymbol{\xi}=0$ with $\nabla_{\mathbf{Y}}$ and use the definition of the Riemann curvature tensor and of the torsion, which vanishes identically, to obtain the Jacobi equation

$$
\nabla_{\boldsymbol{\xi}} \nabla_{\boldsymbol{\xi}} \mathbf{Y}+\mathbf{R}(\mathbf{Y}, \boldsymbol{\xi}) \boldsymbol{\xi}=0
$$

Here, as is well known, the torsion tensor and the Riemann curvature tensor are defined, respectively, to be

$$
\begin{gathered}
\mathbf{T}(\mathbf{Y}, \mathbf{Z})=\nabla_{\mathbf{Y}} \mathbf{Z}-\nabla_{\mathbf{Z}} \mathbf{Y}-[\mathbf{Y}, \mathbf{Z}], \\
\mathbf{R}(\mathbf{Y}, \mathbf{Z}) \mathbf{W}=\nabla_{\mathbf{Y}} \nabla_{\mathbf{Z}} \mathbf{W}-\nabla_{\mathbf{Z}} \nabla_{\mathbf{Y}} \mathbf{W}-\nabla_{[\mathbf{Y}, \mathbf{Z}]} \mathbf{W},
\end{gathered}
$$

where $\mathbf{Y}, \mathbf{Z}, \mathbf{W} \in \bar{X}(M)$, and the Riemann curvature tensor has symmetries such as

$$
\begin{aligned}
\mathbf{g}(\mathbf{R}(\mathbf{Y}, \mathbf{Z}) \mathbf{W}, \mathbf{U}) & =-\mathbf{g}(\mathbf{R}(\mathbf{Z}, \mathbf{Y}) \mathbf{W}, \mathbf{U})=-\mathbf{g}(\mathbf{R}(\mathbf{Y}, \mathbf{Z}) \mathbf{U}, \mathbf{W}) \\
& =\mathbf{g}(\mathbf{R}(\mathbf{W}, \mathbf{U}) \mathbf{Y}, \mathbf{Z}),
\end{aligned}
$$

where $\mathbf{Y}, \mathbf{Z}, \mathbf{W}, \mathbf{U} \in \bar{X}(M)$. Local components of the Riemann curvature tensor are expressed as

$$
\begin{gathered}
R_{i j k \ell}=R_{i j k}^{m} g_{m \ell}=\mathbf{g}\left(\mathbf{R}\left(\frac{\partial}{\partial x^{i}}, \frac{\partial}{\partial x^{j}}\right) \frac{\partial}{\partial x^{k}}, \frac{\partial}{\partial x^{\ell}}\right), \\
R_{i j k}^{\ell}=\frac{\partial \Gamma_{j k}^{\ell}}{\partial x^{i}}-\frac{\partial \Gamma_{i k}^{\ell}}{\partial x^{j}}+\Gamma_{i m}^{\ell} \Gamma_{j k}^{m}-\Gamma_{j m}^{\ell} \Gamma_{i k}^{m} .
\end{gathered}
$$

In the next section, we will give an example of Riemannian metrics whose geodesics are equivalent to trajectories of Newton's equations of motion

$$
\frac{\mathrm{d}^{2} q^{i}}{\mathrm{~d} t^{2}}+\frac{\partial V}{\partial q^{i}}=0, \quad i=1, \ldots, N
$$

Then, in order to analyze stability/instability of trajectories of the natural Hamiltonian system, we can deal with the Jacobi equation, a linearization of the geodesic equation. However, the Jacobi equations in their original form are not suitable to Lyapunov analysis.

\section{B. Geodesics for the Jacobi metric}

Consider equations of motion, Eq. (14), on $\mathbf{R}^{N}$, which we call a natural dynamical system. Let $M_{J}$ be an open submanifold of the configuration space $\mathbf{R}^{N}$, which is defined to be

$$
M_{J}=\left\{q \in \mathbf{R}^{N} \mid V(q)<E\right\} .
$$


As is well known, if energy is fixed at $E$, almost all trajectories are confined in $M_{J}$ when $N \geqslant 2$. On the other hand, the Jacobi metric $\mathbf{g}_{J}$ is defined, in $M_{J}$, to be

$$
\left(g_{J}\right)_{i j}=2[E-V(q)] \delta_{i j} .
$$

According to Maupertuis's Principle of Least Action, an extremal of the action, the integral of the kinetic energy along possible paths, provides an actual trajectory of total-energy $E$. This principle can also be stated as follows: An extremal of the variational problem of lengths of paths with respect to the Jacobi metric provides an actual trajectory of the total energy $E[12,13]$. From Eq. (10) along with the Jacobi metric $\mathbf{g}_{J}$, the arc length parameter $s$ is shown to be related to the time parameter $t$ by

$$
\mathrm{d} s^{2}=4[E-V(q)]^{2} \mathrm{~d} t^{2},
$$

and the tangent vector to a geodesic is always unity accordingly

$$
\begin{aligned}
\mathbf{g}_{J}(\boldsymbol{\xi}, \boldsymbol{\xi}) & =g_{i j} \xi^{i} \xi^{j}=2(E-V) \delta_{i j} \frac{\mathrm{d} q^{i}}{\mathrm{~d} s} \frac{\mathrm{d} q^{j}}{\mathrm{~d} s}=[2(E-V)]^{2}\left(\frac{\mathrm{d} t}{\mathrm{~d} s}\right)^{2} \\
& =1 .
\end{aligned}
$$

Since the Christoffel symbols for the Jacobi metric (16) are given by

$$
\Gamma_{j k}^{i}=\frac{-1}{2(E-V)}\left[\frac{\partial V}{\partial q^{j}} \delta_{k}^{i}+\frac{\partial V}{\partial q^{k}} \delta_{j}^{i}-\frac{\partial V}{\partial q^{i}} \delta_{j k}\right],
$$

the geodesic equations for the Jacobi metric are expressed as

$$
\frac{\mathrm{d}^{2} q^{i}}{\mathrm{~d} s^{2}}-\frac{1}{E-V} \frac{\partial V}{\partial q^{j}} \frac{\mathrm{d} q^{j}}{\mathrm{~d} s} \frac{\mathrm{d} q^{i}}{\mathrm{~d} s}+\frac{1}{4(E-V)^{2}} \frac{\partial V}{\partial q^{i}}=0,
$$

which prove to be equivalent to Newton's equations of motion (14) on account of Eq. (17). However, the Jacobi Eqs. (6) with the curvature tensor for the Jacobi metric are not brought into the same equations as Eq. (8), a linearization of Newton's equations of motion, in general. Components of the curvature tensor for $\mathbf{g}_{J}$ are indeed put in the form $[11,13]$

$$
R_{i j k \ell}=C_{i \ell} \delta_{j k}+C_{j k} \delta_{i \ell}-C_{i k} \delta_{j \ell}-C_{j \ell} \delta_{i k},
$$

where

$$
\begin{aligned}
C_{i j}= & \frac{\partial^{2} V}{\partial q^{i} \partial q^{j}}+\frac{3}{2(E-V)} \frac{\partial V}{\partial q^{i}} \frac{\partial V}{\partial q^{j}} \\
& -\frac{1}{4(E-V)} \delta^{k \ell} \frac{\partial V}{\partial q^{k}} \frac{\partial V}{\partial q^{\ell}} \delta_{i j} .
\end{aligned}
$$

\section{GEODESIC FLOWS ON COTANGENT BUNDLES}

In the previous section, we have mentioned that trajectories of a natural dynamical system with a fixed energy may be regarded as geodesics on a suitable Riemannian manifold, and that stability/instability of the trajectories are analyzed through the Jacobi equation, a linearization of the geodesic equation. However, the Jacobi equation is a second-order differential equation, while Lyapunov analysis is applied to first-order differential equations. We hence need a first-order differential equation associated with the Jacobi equation in order to apply Lyapunov analysis. To find such a first-order differential equation, we are working on the cotangent bundle $T^{*} M$ of a Riemannian manifold $M$ along with some geometric setting ups on $T^{*} M$. Related topics on $T^{*} M$ will be described in Appendix A.

At first, let us be reminded of a minimum on cotangent bundles. Let $M$ be an $m$-dimensional Riemannian manifold endowed with Riemannian metric $\mathbf{g}=g_{i j} \mathrm{~d} x^{i} \otimes \mathrm{d} x^{j}$, and $T^{*} M$ the cotangent bundle of $M$ with the projection $\pi: T^{*} M \rightarrow M$. Let $\left(x^{i}\right)$ and $\left(x^{i}, p_{i}\right)$ be local coordinates in an open subset $U \subset M$ and in $\pi^{-1}(U)$, respectively. Further, let $\left(\bar{x}^{i}, \bar{p}_{i}\right)$ be another local coordinates in $\pi^{-1}(\bar{U})$ with $\pi^{-1}(U) \cap \pi^{-1}(\bar{U}) \neq \varnothing$. Then one has the coordinate transformation in the intersection $\pi^{-1}(U) \cap \pi^{-1}(\bar{U})$,

$$
\bar{x}^{i}=\bar{x}^{i}(x), \quad \bar{p}_{i}=\frac{\partial x^{j}}{\partial \bar{x}^{i}} p_{j} .
$$

\section{A. Geodesic flows}

We recall that Newton's equations of motion have been already "geometrized" so as to be geodesic equations on a suitable Riemannian manifold, so that further external force does not need to be taken into account anymore. In other words, we have only to consider a free particle motion on $M$. In the Hamiltonian formalism, the Hamiltonian we then have to study should be given, on $T^{*} M$, by

$$
K=\frac{1}{2} g^{i j}(x) p_{i} p_{j},
$$

where $\left(g^{i j}\right):=\left(g_{i j}\right)^{-1}$. Hamilton's equations of motion for $K$ are then put in the form

$$
\frac{\mathrm{d} x^{i}}{\mathrm{~d} s}=\frac{\partial K}{\partial p_{i}}=g^{i j} p_{j}
$$

$$
\frac{\mathrm{d} p_{i}}{\mathrm{~d} s}=-\frac{\partial K}{\partial x^{i}}=g^{k j} \Gamma_{j i}^{\ell} p_{k} p_{\ell},
$$

where use has been made of the equality

$$
-\frac{\partial g^{k \ell}}{\partial x^{i}}=g^{k j} \Gamma_{j i}^{\ell}+g^{j \ell} \Gamma_{j i}^{k}
$$

It is an easy matter to show that Eq. (21) projects to geodesic equation on $M$. In fact, put together, differentiation of the first equation of Eq. (21) with respect to $s$ and the second equation of Eq. (21) along with the above equality provide geodesic equations. As is well known, Eq. (21) is associated with the Hamiltonian vector field $\mathbf{X}_{K}$ given by 


$$
\mathbf{X}_{K}=\frac{\partial K}{\partial p_{i}} \frac{\partial}{\partial x^{i}}-\frac{\partial K}{\partial x^{i}} \frac{\partial}{\partial p_{i}}=g^{i j} p_{j} \frac{\partial}{\partial x^{i}}+g^{k j} \Gamma_{j i}^{\ell} p_{k} p_{\ell} \frac{\partial}{\partial p_{i}} .
$$

Integral curves of Eq. (22) are called geodesic flows. We note here that the nomenclature "geodesic flows" are usually assigned to the corresponding flows on the tangent bundle, but we use the word for convenience's sake.

It is worth noting here that how geodesic flows on $T^{*} M$ project to geodesics on $M$. Let $P(s)=(x(s), p(s))$ be a geodesic flow with an initial value $(x(0), p(0))=(a, b)$ with $g^{i j} b_{i} b_{j}=1$. Define a tangent vector $v$ to $M$ at $a=\pi(P(0))$ by $v^{i}=g^{i j} b_{j}$. Then the projection $x(s)=\pi(P(s))$ is a geodesic with the initial value $(x(0), \dot{x}(0))=(a, v)$. Varying $b$ $\in T_{a}^{*} M$ with $g^{i j} b_{i} b_{j}=1$ but fixing $a$, we obtain an $(m-1)$-parameter family of geodesic flows on $T^{*} M$, which projects to an $(m-1)$-parameter family of geodesics passing the point $a$ of $M$. Furthermore, the vector field $\mathbf{X}_{K}$ projects to the tangent vector field $\boldsymbol{\xi}$ to this family of geodesics $M$. However, $\boldsymbol{\xi}$ has singularity only at $a \in M$ in a neighborhood of $a$. We have to note that if all geodesic flows on $T^{*} M$ project to geodesics $M$, those geodesics may not define such a tangent vector field uniquely. If there is another $(m-1)$-parameter family of geodesic flows on $T^{*} M$, it may project to another $(m-1)$-parameter family of geodesics on $M$ along with a tangent vector field like $\boldsymbol{\xi}$.

\section{B. Adapted frames}

To describe geodesic flows in a more geometric way, we introduce an adapted frame and an adapted coframe on $\pi^{-1}(U) \subset T^{*} M$ by the use of the Christoffel symbols $\Gamma_{j k}^{i}$ on $M$. The adapted frame and coframe are defined, in $\pi^{-1}(U)$, to be

$$
D_{i}=\frac{\partial}{\partial x^{i}}+p_{k} \Gamma_{i j}^{k} \frac{\partial}{\partial p_{j}}, \quad D_{i}^{-}=\frac{\partial}{\partial p_{i}},
$$

and

$$
\theta^{i}=\mathrm{d} x^{i}, \quad \theta^{\bar{i}}=\mathrm{d} p_{i}-p_{k} \Gamma_{i j}^{k} \mathrm{~d} x^{j},
$$

respectively, where $\bar{i}=i+m$. These frames are dual to each other, i.e., they satisfy

$$
\theta^{i}\left(D_{j}\right)=\delta_{j}^{i}, \quad \theta^{i}\left(D_{\bar{j}}\right)=0, \quad \theta^{\bar{i}}\left(D_{j}\right)=0, \quad \theta^{\bar{i}}\left(D_{\bar{j}}\right)=\delta_{i}^{j} .
$$

If there is another adapted frame $\left\{\bar{D}_{i}, \bar{D}_{\bar{i}}\right\}$ in an open set $\pi^{-1}(\bar{U})$ and if the intersection $\pi^{-1}(\bar{U}) \cap \pi^{-1}(U)$ is nonempty, then from Eq. (19) it follows that the adapted frames are subject to the transformation

$$
\bar{D}_{i}=\frac{\partial x^{j}}{\partial \bar{x}^{i}} D_{j}, \quad \bar{D}_{\bar{i}}=\frac{\partial \bar{x}^{i}}{\partial x^{j}} D_{\bar{j}} .
$$

For adapted coframes, an analogous transformation holds as well.
The transformation (25) implies that $D_{i}, i=1, \ldots, m$, and $D_{\bar{i}}, \bar{i}=m+1, \ldots, 2 m$, define, respectively, subspaces $H_{P}$ and $V_{P}$ of the tangent space $T_{P} T^{*} M$ at each point $P$ $\in T^{*} M$ independently of the choice of adapted frames. Thus one obtains a direct sum decomposition of the tangent space to $T^{*} M$ at each point $P \in T^{*} M$,

$$
T_{P} T^{*} M=H_{P} \oplus V_{P} .
$$

The subspaces $H_{P}$ and $V_{P}$ are called the horizontal and the vertical subspace of $T_{P} T^{*} M$, respectively. We notice here that $H_{P}$ and $T_{\pi(P)} M$ are isomorphic as vector spaces. Note further that the transformation rule for the standard frame $\left\{\partial / \partial x^{i}, \partial / \partial p_{i}\right\}$ is mixed up, so that one cannot define a subspace, say, $\operatorname{span}\left\{\partial / \partial x^{i}\right\}$ independently of the choice of natural frames. See [14] for adapted frames on the tangent bundle $T M$.

In terms of the adapted frame, the Hamiltonian vector field $\mathbf{X}_{K}$ becomes expressed as

$$
\mathbf{X}_{K}=D_{\bar{i}}(K) D_{i}-D_{i}(K) D_{\bar{i}}^{-}=g^{i j} p_{j} D_{i},
$$

which shows that $\mathbf{X}_{K}$ is a horizontal vector field and further that geodesic flows are horizontal curves in the sense that the tangent vectors to them are always horizontal.

\section{The Sasaki metric}

As is already seen, the tangent space to $T^{*} M$ at each point of $T^{*} M$ is decomposed into a direct sum. We may define a metric on $T^{*} M$ so that the decomposition may be orthogonal direct sum. One of such metrics is the Sasaki metric, which is a lifted metric $\tilde{\mathbf{g}}$ given by

$$
\tilde{\mathbf{g}}=g_{i j} \theta^{i} \otimes \theta^{j}+g^{i j} \theta^{\bar{i}} \otimes \theta^{\bar{j}} .
$$

This metric is defined independently of the choice of adapted coframes. We notice here that the Sasaki metric was introduced on the tangent bundle $T M$ [15], but we use the same nomenclature on the cotangent bundle $T^{*} M$ as well.

By using the Sasaki metric, the arc length on $T^{*} M$ is defined as

$$
\mathrm{d} \sigma^{2}=g_{i j} \mathrm{~d} x^{i} \mathrm{~d} x^{j}+g^{i j}\left(\mathrm{~d} p_{i}-p_{k} \Gamma_{i n}^{k} \mathrm{~d} x^{n}\right)\left(\mathrm{d} p_{j}-p_{\ell} \Gamma_{j h}^{\ell} \mathrm{d} x^{h}\right) .
$$

It then turns out that geodesic flows on $T^{*} M$ take the same arc length as the corresponding geodesics on $M$ have, since one has $\mathrm{d} \sigma^{2}=g_{i j} \mathrm{~d} x^{i} \mathrm{~d} x^{j}=\mathrm{d} s^{2}$ for horizontal curves, and since geodesic flows are horizontal. Hence, the parameter $s$ used in Hamilton's Eq. (21) may be interpreted as the arc length on $M$, so that the geodesic $x(s)=\pi(P(s))$ on $M$ is described in the arc length parameter.

We will adopt the Sasaki metric on $T^{*} M$ to discuss orthogonality of Lyapunov vectors on $T^{*} M$ in the next section.

\section{LYAPUNOV ANALYSIS OF GEODESIC FLOWS}

On the basis of the geometric setting up, we are to find a first-order differential equation associated with the Jacobi equation, and thereby discuss Lyapunov vectors. 


\section{A. Linearization of Hamilton's equations of motion}

For a general Hamiltonian function $H$, linearized Hamilton's equations of motion are put, as is well known, in the form

$$
\begin{gathered}
\frac{\mathrm{d} \hat{X}^{i}}{\mathrm{~d} s}=\frac{\partial^{2} H}{\partial p_{i} \partial x^{j}} \hat{X}^{j}+\frac{\partial^{2} H}{\partial p_{i} \partial p_{j}} \hat{X}^{\bar{j}}, \\
\frac{\mathrm{d} \hat{X}^{\bar{i}}}{\mathrm{~d} s}=-\frac{\partial^{2} H}{\partial x^{i} \partial x^{j}} \hat{X}^{j}-\frac{\partial^{2} H}{\partial x^{i} \partial p_{j}} \hat{X}^{\bar{j}},
\end{gathered}
$$

where $\mathbf{X}=\hat{X}^{i} \partial_{i}+\hat{X}^{\bar{i}} \partial_{\bar{i}}$ stands for a deviation of Hamiltonian flows, where $\partial_{i}=\partial / \partial x^{i}$ and $\partial_{i}=\partial / \partial p_{i}$. These equations may be obtained from the condition $\left[\mathbf{X}, \mathbf{X}_{H}\right]=0$ as well, where $\mathbf{X}_{H}$ is the Hamiltonian vector field,

$$
\mathbf{X}_{H}=\frac{\partial H}{\partial p_{i}} \frac{\partial}{\partial x^{i}}-\frac{\partial H}{\partial x^{i}} \frac{\partial}{\partial p_{i}}
$$

In fact, the condition $\left[\mathbf{X}, \mathbf{X}_{H}\right]=0$ restricted to a prescribed Hamiltonian flow $P(s)=(x(s), p(s))$ provides

$$
\begin{aligned}
{\left.\left[\mathbf{X}, \mathbf{X}_{H}\right]\right|_{P(s)}=} & \left.\left(\frac{\partial^{2} H}{\partial p_{i} \partial x^{j}} \hat{X}^{j}+\frac{\partial^{2} H}{\partial p_{i} \partial p_{j}} \hat{X}^{\bar{j}}\right) \frac{\partial}{\partial x^{i}}\right|_{P(s)} \\
& -\left.\left(\frac{\partial^{2} H}{\partial x^{i} \partial x^{j}} \hat{X}^{j}+\frac{\partial^{2} H}{\partial x^{i} \partial p_{j}} \hat{X}^{\bar{j}}\right) \frac{\partial}{\partial p_{i}}\right|_{P(s)} \\
& -\left.\frac{\mathrm{d} \hat{X}^{i}}{\mathrm{~d} s} \frac{\partial}{\partial x^{i}}\right|_{P(s)}-\left.\frac{\mathrm{d} \hat{X}^{\bar{i}}}{\mathrm{~d} s} \frac{\partial}{\partial p_{i}}\right|_{P(s)},
\end{aligned}
$$

where we have used the formula

$$
\frac{\mathrm{d} \hat{X}^{i}}{\mathrm{~d} s}=\frac{\partial H}{\partial p_{k}} \frac{\partial \hat{X}^{i}}{\partial x^{k}}-\frac{\partial H}{\partial x^{k}} \frac{\partial \hat{X}^{i}}{\partial p_{k}}=\mathbf{X}_{H}\left(\hat{X}^{i}\right),
$$

and a similar formula for $\mathrm{d} \hat{X}^{\bar{i}} / \mathrm{d} s$. It is to be noted here that the condition $\left[\mathbf{X}, \mathbf{X}_{H}\right]=0$ implies that a Hamiltonian flow, an integral curve of $\mathbf{X}_{H}$, is dragged to another infinitesimally nearby Hamiltonian flow by the infinitesimal transformation $\mathbf{X}$, i.e., $\mathbf{X}$ is a deviation of Hamiltonian flows. With this in mind, we may obtain linearized equations with respect to the adapted frame, if we calculate $\left[\mathbf{X}, \mathbf{X}_{H}\right]=0$ with $\mathbf{X}$ and $\mathbf{X}_{H}$ expressed as

$$
\begin{gathered}
\mathbf{X}=X^{i} D_{i}+X^{\bar{i}} D_{\bar{i}}, \\
\mathbf{X}_{H}=D_{\bar{i}}(H) D_{i}-D_{i}(H) D_{\bar{i}},
\end{gathered}
$$

respectively, and restrict the resultant equation to a prescribed flow $P(s)$. We note here that the components $\left(X^{i}, X^{\bar{i}}\right)$ with respect to the adapted frame transform according to

$$
X^{i}=\frac{\partial x^{i}}{\partial \bar{x}^{j}} \bar{X}^{j}, \quad X^{\bar{i}}=\frac{\partial \bar{x}^{j}}{\partial x^{i}} \bar{X}^{\bar{j}} .
$$

A long but straightforward calculation of $\left.\left[\mathbf{X}, \mathbf{X}_{H}\right]\right|_{P(s)}=0$ then provides linearized Hamilton's equations of motion as follows:

$$
\begin{aligned}
\frac{\mathrm{d} X^{i}}{\mathrm{~d} s} & =\left[\frac{\partial^{2} H}{\partial p_{i} \partial x^{j}}+\frac{\partial^{2} H}{\partial p_{i} \partial p_{\ell}} p_{k} \Gamma_{\ell j}^{k}\right] X^{j}+\frac{\partial^{2} H}{\partial p_{i} \partial p_{j}} X^{\bar{j}}, \\
\frac{\mathrm{d} X^{\bar{i}}}{\mathrm{~d} s}= & -\left[\frac{\partial^{2} H}{\partial x^{i} \partial x^{j}}+\frac{\partial^{2} H}{\partial x^{i} \partial p_{\ell}} p_{k} \Gamma_{j \ell}^{k}+\left(\frac{\partial^{2} H}{\partial p_{\ell} \partial x^{j}}\right.\right. \\
& \left.\left.+\frac{\partial^{2} H}{\partial p_{\ell} \partial p_{m}} p_{n} \Gamma_{m j}^{n}\right) p_{k} \Gamma_{i \ell}^{k}-\frac{\partial H}{\partial x^{k}} \Gamma_{i j}^{k}+p_{k} \frac{\partial \Gamma_{i j}^{k}}{\partial x^{m}} \frac{\partial H}{\partial p_{m}}\right] X^{j} \\
& -\left[\frac{\partial^{2} H}{\partial x^{i} \partial p_{j}}+\frac{\partial^{2} H}{\partial p_{\ell} \partial p_{j}} p_{k} \Gamma_{i \ell}^{k}\right] X^{\bar{j}},
\end{aligned}
$$

where use has been made of the formula

$$
\frac{\mathrm{d} X^{i}}{\mathrm{~d} s}=D_{\bar{j}}^{-}(H) D_{j}\left(X^{i}\right)-D_{j}(H) D_{j}^{-}\left(X^{i}\right)=\mathbf{X}_{H}\left(X^{i}\right),
$$

and of a similar formula for $\mathrm{d} X^{\bar{i}} / \mathrm{d} s$.

In what follows, we take the Hamiltonian given by Eq. (20). The equation of deviation (30) then takes the form

$$
\begin{gathered}
\frac{\mathrm{d} X^{i}}{\mathrm{~d} s}=-\Gamma_{j k}^{i} g^{k \ell} p_{\ell} X^{j}+g^{i j} X^{\bar{j}}, \\
\frac{\mathrm{d} X^{\bar{i}}}{\mathrm{~d} s}=-R_{j k \ell i} g^{k n} p_{n} g^{\ell h} p_{h} X^{j}+\Gamma_{i k}^{j} g^{k \ell} p_{\ell} X^{\bar{j}} .
\end{gathered}
$$

The right-hand side of Eq. (31) must be evaluated along a geodesic flow $P(s)=(x(s), p(s))$. Since one has $g^{i j} p_{j}(s)$ $=\mathrm{d} x^{i} / \mathrm{d} s=: \xi^{i}(s)$ along the geodesic flow, Eq. (31) can be brought into the form

$$
\begin{gathered}
\frac{\mathrm{d} X^{i}}{\mathrm{~d} s}=-\Gamma_{j k}^{i} \xi^{k} X^{j}+g^{i j} X^{\bar{j}}, \\
\frac{\mathrm{d} X^{\bar{i}}}{\mathrm{~d} s}=-R_{j k \ell i} \xi^{k} \xi^{\ell} X^{j}+\Gamma_{i k}^{j} \xi^{k} X^{\bar{j}} .
\end{gathered}
$$

We can show that this system of equations is the first-order differential equation that project to the Jacobi equation, and hence, may be called the lifted Jacobi equation. The proof runs as follows: On account of Eq. (29), the quantities $\left(X^{i}(s)\right)$ and $\left(X^{\bar{i}}(s)\right)$ may be viewed as a tangent and a cotangent vector to $M$ along the geodesic $x(s)$, so that the first equation of Eq. (32), rewritten as

$$
\frac{\mathrm{d} X^{i}}{\mathrm{~d} s}+\Gamma_{j k}^{i} \xi^{k} X^{j}=g^{i j} X^{\bar{j}},
$$

implies that $\left(g^{i j} X^{\bar{j}}(s)\right)$ is equal to the covariant derivative of $\left(X^{i}(s)\right)$ along the geodesic $x(s)$. The second equation of Eq. (32) then implies that 


$$
\frac{\mathrm{d} Y^{i}}{\mathrm{~d} s}+\Gamma_{j k}^{i} \xi^{k} Y^{j}=-R_{j k} \ell^{i} \xi^{k} \xi^{\ell} X^{j} \quad \text { with } \quad Y^{i}=g^{i j} X^{\bar{j}}
$$

The above two equations are put together to yield the Jacobi equation for $\mathbf{Y}_{\mathbf{X}}=\left(X^{i}(s)\right)$,

$$
\nabla_{\xi} \nabla_{\xi} \mathbf{Y}_{\mathbf{X}}=-\mathbf{R}\left(\mathbf{Y}_{\mathbf{X}}, \boldsymbol{\xi}\right) \boldsymbol{\xi}
$$

where $\nabla_{\xi}$ stands for the covariant derivation along the geodesic $x(s)$.

\section{B. Lyapunov vectors}

Here, we show that solutions to Eq. (32) satisfy the requirement stated in the Introduction in the Hamiltonian system with the Hamiltonian $K$ given in Eq. (20). As for the gradient of $K$, we note that the differential $\mathrm{d} K$ and the gradient of $K, \operatorname{grad} K$, are put in the form

$$
\mathrm{d} K=g^{i k} p_{k} \theta^{\bar{i}}, \quad \operatorname{grad} K=p_{i} D_{\bar{i}}^{\overline{1}},
$$

respectively, where the gradient of a function $F$ on $T^{*} M, \operatorname{grad} F$, is defined through

$$
\widetilde{\mathbf{g}}(\operatorname{grad} F, \mathbf{X})=\mathrm{d} F(\mathbf{X})
$$

for any vector field $\mathbf{X} \in \bar{X}\left(T^{*} M\right)$.

It is an easy matter to verify that Eq. (32) is satisfied by $\mathbf{X}_{K}$, the tangent vector to a Hamiltonian flow $P(s)$ or a geodesic flow in $T^{*} M$. In fact, the tangent vector $\mathbf{X}_{K}$ to $P(s)$ is given Eq. (27), and has the components, $X^{i}(s)$ $=g^{i \ell} p_{\ell}(s), X^{\bar{i}}(s)=0$, satisfying Eq. (32). While the gradient vector along the Hamiltonian flow $P(s)$, which is denoted by $\operatorname{grad} K(s)$ for simplicity, is not a solution to the linearized Eq. (32), the vector $\operatorname{grad} K(s)+s \mathbf{X}_{K}(s)=p_{i}(s) D_{\bar{i}}$ $+s g^{i k} p_{k}(s) D_{i}$ is a solution to Eq. (32), as is easily verified. Taking this into account, we wish to decompose the tangent space $T_{P(s)} T^{*} M$ to $T^{*} M$ at every point $P(s)$ of a geodesic flow into the direct sum of the plane spanned by both $\mathbf{X}_{K}(s)$ and $\operatorname{grad} K(s)$ and the subspace transversal to the plane. Let us define subspaces $N_{P(s)}$ and $E_{P(s)}$ to be

$$
\begin{gathered}
N_{P(s)}=\left\{\mathbf{X} \in T_{P(s)} T^{*} M \mid \mathbf{X}=\alpha \mathbf{X}_{K}(s)+\beta \operatorname{grad} K(s),\right. \\
\alpha, \beta \in \mathbf{R}\}, \\
E_{P(s)}=\left\{\mathbf{X} \in T_{P(s)} T^{*} M \mid \tilde{\mathbf{g}}\left(\mathbf{X}, \mathbf{X}_{K}(s)\right)=0,\right. \\
\tilde{\mathbf{g}}(\mathbf{X}, \operatorname{grad} K(s))=0\},
\end{gathered}
$$

respectively, where $E_{P(s)}$ is the orthogonal complement of $N_{P(s)}$ with respect to the Sasaki metric $\widetilde{\mathbf{g}}$. Thus, we have the orthogonal direct sum decomposition,

$$
T_{P(s)} T^{*} M=N_{p(s)} \oplus E_{P(s)} .
$$

We wish to show that these subspaces are invariant under any solution to the linearized equation (32). To this end, we have to verify,
Theorem. A solution $\mathbf{X}(s)$ to the linearized Eq. (32) which is in $N_{P(0)}$ (resp. in $E_{P(0)}$ ) at an initial moment $s$ $=0$ keeps belonging to $N_{P(s)}$ (resp. to $E_{P(s)}$ ) at any instant $s$.

The proof of this statement is carried out as follows: As we have already shown, $\mathbf{X}_{K}(s)$ and $\operatorname{grad} K(s)+s \mathbf{X}_{K}(s)$ are solutions to Eq. (32), so that the linear combination of them, $\alpha \mathbf{X}_{K}(s)+\beta\left(\operatorname{grad} K(s)+s \mathbf{X}_{K}(s)\right)=(\alpha+\beta s) \mathbf{X}_{K}(s)$ $+\beta \operatorname{grad} K(s)$, is also in $N_{P(s)}$ at any instant $s$, which proves the invariance of $N_{P(s)}$ under the linearized flow $\mathbf{X}(s)$. To prove the invariance of $E_{P(s)}$, we consider the temporal evolution of $\tilde{\mathbf{g}}\left(\mathbf{X}, \mathbf{X}_{K}\right)$ with $\mathbf{X}$ a solution to Eq. (32). We are to show that

$$
\begin{gathered}
\frac{\mathrm{d}}{\mathrm{d} s} \tilde{\mathbf{g}}\left(\mathbf{X}, \mathbf{X}_{K}\right)=\mathrm{d} K(\mathbf{X}), \\
\frac{\mathrm{d}^{2}}{\mathrm{~d} s^{2}} \tilde{\mathbf{g}}\left(\mathbf{X}, \mathbf{X}_{K}\right)=0 .
\end{gathered}
$$

We can carry out the proof of these equations in the manner of mechanics as follows: Note that $\tilde{\mathbf{g}}\left(\mathbf{X}, \mathbf{X}_{K}\right)=\theta(\mathbf{X})$, where $\theta$ is the standard one form on $T^{*} M$, i.e., $\theta=p_{i} \mathrm{~d} x^{i}$ in local coordinates. Then differentiation of $\theta(\mathbf{X})$ with respect to $s$ results in

$$
\begin{aligned}
\frac{\mathrm{d}}{\mathrm{d} s} \theta(\mathbf{X}) & =\mathcal{L}_{\mathbf{X}_{K}}(\theta(\mathbf{X}))=\left(\mathcal{L}_{\mathbf{X}_{K}} \theta\right)(\mathbf{X})+\theta\left(\left[\mathbf{X}_{K}, \mathbf{X}\right]\right) \\
& =\left[\mathrm{d} \iota\left(\mathbf{X}_{K}\right) \theta+\iota\left(\mathbf{X}_{K}\right) \mathrm{d} \theta\right](\mathbf{X})=\left[\mathrm{d}\left(\theta\left(\mathbf{X}_{K}\right)\right)-\mathrm{d} K\right](\mathbf{X}) \\
& =\mathrm{d} K(\mathbf{X}),
\end{aligned}
$$

where use has been made of (i) the definition of the Lie derivative of one forms, (ii) the condition $\left[\mathbf{X}, \mathbf{X}_{K}\right]=0$, (iii) the Cartan's formula for the Lie derivation, (iv) $\iota\left(\mathbf{X}_{K}\right) \mathrm{d} \theta$ $=-\mathrm{d} K$, and $(\mathrm{v})$ the equality $\theta\left(\mathbf{X}_{K}\right)=2 K$ due to the homogeneity of $K$ in $p_{i}$. Thus, we obtain the former equation of Eq. (35). Differentiating the former equation of Eq. (35) with respect to $s$ using the equation

$$
\frac{\mathrm{d}}{\mathrm{d} s} \mathrm{~d} K(\mathbf{X})=\frac{\mathrm{d}}{\mathrm{ds}} \tilde{\mathbf{g}}(\operatorname{grad} K, \mathbf{X})=0,
$$

a similar equation to Eq. (5), we obtain the latter of Eq. (35). Now, Eq. (35) is integrated to give

$$
\left.\tilde{\mathbf{g}}\left(\mathbf{X}, \mathbf{X}_{K}\right)\right|_{P(s)}=\left.\tilde{\mathbf{g}}\left(\mathbf{X}, \mathbf{X}_{K}\right)\right|_{P(0)}+\left.s \mathrm{~d} K(\mathbf{X})\right|_{P(0)} .
$$

Since $\mathrm{d} K(\mathbf{X})=\tilde{\mathbf{g}}(\mathbf{X}, \operatorname{grad} K)$, the above equation implies that $\mathbf{X}(s) \in E_{P(s)}$ if $\mathbf{X}(0) \in E_{P(0)}$. This ends the proof of the invariance of $E_{P(s)}$ under the linearized flow $\mathbf{X}(s)$.

On the basis of the decomposition (34), we can construct a set of Lyapunov vectors $\left\{\mathbf{V}_{a}\right\}, a=1, \ldots, 2 m$, satisfying the requirement mentioned in Sec. I. We are thinking of the Riemannian manifold $\left(M_{J}, \mathbf{g}_{J}\right)$ introduced in Sec. II B, and hence, $m=N$. The first $N-1$ linearly independent solutions, $\left\{\mathbf{X}_{a}(s)\right\}, a=1, \ldots, N-1$, to the lifted Jacobi Eq. (32) are chosen in $E_{P(s)}$, which are orthogonalized to give first $N$ -1 Lyapunov vectors $\left\{\mathbf{V}_{1}, \ldots, \mathbf{V}_{N-1}\right\}$. The $N$ th and $(N$ 
TABLE I. Comparison between the usual method and the geometric method. The $N$-dimensional manifold $M_{J}$ is defined in Eq. (15), and $\tilde{\mathbf{g}}_{E}$ is the Euclidean metric. Note that $T^{*} M_{J}=M_{J} \times \mathbf{R}^{N}$.

\begin{tabular}{lccccc}
\hline \hline Method & $\begin{array}{c}\text { Configuration } \\
\text { space }\end{array}$ & $\begin{array}{c}\text { Phase } \\
\text { space }\end{array}$ & Metric & Hamiltonian & $\begin{array}{c}\text { Linearized } \\
\text { equation }\end{array}$ \\
\hline Usual & $M_{J}$ & $M_{J} \times \mathbf{R}^{N}$ & $\tilde{\mathbf{g}}_{E}$ & $H$ [Eq. (1)] & Eq. (2) \\
Geometric & $M_{J}$ & $T^{*} M_{J}$ & $\tilde{\mathbf{g}}_{J}$ & $K$ [Eq. (20)] & Eq. (32) \\
\hline \hline
\end{tabular}

$+1)$-th Lyapunov vectors are chosen in $N_{P(s)}$ so as to be $\mathbf{V}_{N}(s)=\mathbf{X}_{K}(s)$ and $\mathbf{V}_{N+1}(s)=\operatorname{grad} K(s)$, respectively. This is because they are mutual orthogonal and because $\mathbf{X}_{K}(s)$ and $\operatorname{grad} K(s)+s \mathbf{X}_{K}(s)$ are solutions to the linearized equation and further orthogonal to the first $N-1$ Lyapunov vectors. Note in addition that any solution $\mathbf{X}(s)$ staying in $N_{P(s)}$ becomes asymptotically parallel to $\mathbf{X}_{K}(s)$ as $s \rightarrow \infty$, so that $\mathbf{X}_{K}(s)$ is assigned to the $N$ th Lyapunov vector and $\operatorname{grad} H(s)$ to the $(N+1)$-th Lyapunov vector, respectively. The remaining $N-1$ Lyapunov vectors are chosen in $E_{P(s)}$, which are orthogonal to $\mathbf{X}_{K}$ and $\operatorname{grad} K$ as well as to the first $N-1$ Lyapunov vectors by the very definition. Consequently, from solutions to Eq. (32) with the initial values chosen so as to satisfy

(a) $\mathbf{X}_{N}(0)=\mathbf{X}_{K}(0), \quad \mathbf{X}_{N+1}(0)=\operatorname{grad} K(0)$,

(b) $\mathbf{X}_{a}(0) \perp\left\{\mathbf{X}_{K}(s), \quad \operatorname{grad} K(0)\right\}, \quad a=1, \ldots, \quad N-1, N$ $+2, \ldots, 2 N$,

at the initial moment $s=0$, we may obtain expectedly a set of Lyapunov vectors such that

(i) $\mathbf{V}_{N}(s)=\mathbf{X}_{K}(s), \quad \mathbf{V}_{N+1}(s)=\operatorname{grad} K(s)$,

(ii) $\mathbf{V}_{a}(s) \perp\left\{\mathbf{X}_{K}(s), \quad \operatorname{grad} K(s)\right\}, \quad a=1, \ldots, N-1, N$ $+2, \ldots, 2 N$.

From the property (i), we may observe that the Lyapunov exponents $\lambda_{N}$ and $\lambda_{N+1}$ vanish indeed. In fact, since

$$
\widetilde{\mathbf{g}}\left(\mathbf{X}_{K}, \mathbf{X}_{K}\right)=\tilde{\mathbf{g}}(\operatorname{grad} K, \operatorname{grad} K)=2 K
$$

is constant along any geodesic flow, one has $\lambda_{N}=\lambda_{N+1}=0$ from the formula (4).

\section{NUMERICAL CALCULATIONS FOR COMPARISON}

In this section, we are to compare the geometric method and the usual method through a model system with three degrees of freedom, by numerically calculating Lyapunov exponents and Lyapunov vectors in respective methods. We will find that the Lyapunov exponents calculated in respective methods coincide with each other, independently of the choice of methods, while the Lyapunov vectors calculated on respective setting ups exhibit different behaviors to each other, depending on the method chosen.

\section{A. Comparison of setting ups in respective methods}

For a natural Hamiltonian system with $N$ degrees of freedom, setting ups for Lyapunov analysis both in the geometric method and in the usual method are summarized in Table I. We note here that the metric $\tilde{\mathbf{g}}_{E}$ introduced on the phase space $M_{J} \times \mathbf{R}^{N}$ in the usual method is, of course, the Euclidean metric defined, as usual, to be

$$
\widetilde{\mathbf{g}}_{E}=\delta_{i j} \mathrm{~d} q^{i} \otimes \mathrm{d} q^{j}+\delta^{i j} \mathrm{~d} p_{i} \otimes \mathrm{d} p_{j} .
$$

As was pointed out in Sec. II B, the geodesic equations for the Jacobi metric are equivalent to Newton's equations of motion for a natural dynamical system with energy $E$. We now verify this fact in the Hamiltonian formalism. The Hamiltonian vector fields $\mathbf{X}_{K}$ and $\mathbf{X}_{H}$, which are defined on the same phase space in respective manners, are given by

$$
\mathbf{X}_{K}=g^{i j} p_{j} D_{i}, \quad \mathbf{X}_{H}=\delta^{i j}\left(p_{j} \frac{\partial}{\partial q^{i}}-\frac{\partial V}{\partial q^{j}} \frac{\partial}{\partial p_{i}}\right),
$$

respectively, where $g^{i j}=\delta^{i j} /[2(E-V)]$. A straightforward calculation along with Eq. (18) and $\frac{1}{2} \sum_{i} p_{i}^{2}+V=E$ then provides

$$
\mathbf{X}_{K}=\frac{1}{2(E-V)} \mathbf{X}_{H},
$$

which implies that Hamiltonian flows both in the geometric method and in the usual method coincide within the change of parameters, $\mathrm{d} s / \mathrm{d} t=2(E-V(q))$. Thus, along the same flow (up to the parameter change), we may compare numerically tangent vectors such as solutions to linearized equations of motion and Lyapunov vectors. In the following, $\mathbf{X}^{(g)}(s)$ and $\mathbf{X}(t)$ denote solutions to the linearized equations of motion in the geometric method and in the usual method, respectively.

\section{B. Orthogonal relations in the usual method}

In Sec. IV, we have shown that Lyapunov vectors in the geometric method may be chosen so that two of them may be the tangent vectors to the Hamiltonian flow in question and the gradient vector of the Hamiltonian function along the flow, and the others be orthogonal to those two vectors. In this section, we remark that such orthogonal relations holds for part of Lyapunov vectors even in the usual method, in which the Euclidean metric $\widetilde{\mathbf{g}}_{E}$ is adopted in $M_{J} \times \mathbf{R}^{N}$.

Let $\mathbf{X}_{1}(t), \ldots, \mathbf{X}_{2 N}(t)$ be linearly independent solutions to Eq. (2), for which the initial conditions are taken in such a manner that

(a) $\mathbf{X}_{N}(0)=\mathbf{X}_{H}(0), \quad \mathbf{X}_{N+1}(0)=\operatorname{grad} H(0)$,

(b) $\mathbf{X}_{a}(0) \perp\left\{\mathbf{X}_{H}(0), \operatorname{grad} H(0)\right\}$,

$a=1, \ldots, N-1, N+2, \ldots, 2 N$,

where $\mathbf{X}_{H}$ and $\operatorname{grad} H$ are the Hamiltonian vector field for $H$ and the gradient vector field of $H$, respectively. Let $\mathbf{V}_{1}(t), \ldots, \mathbf{V}_{2 N}(t)$ be Lyapunov vectors formed from $\mathbf{X}_{a}(t), a=1, \ldots, 2 N$. Then the following two properties hold true: 
(i) $\mathbf{V}_{1}(t), \ldots, \mathbf{V}_{N}(t)$ are always orthogonal to $\operatorname{grad} H(t)$,

(ii) $\mathbf{V}_{N+1}(t), \ldots, \mathbf{V}_{2 N}(t)$ are always orthogonal to $\mathbf{X}_{H}(t)$.

The property (i) is easily shown to hold from Eq. (5). In fact, solutions $\mathbf{X}_{1}(t), \ldots, \mathbf{X}_{N}(t)$ to the linearized Eqs. (2) are always orthogonal to $\operatorname{grad} H(t)$, if they are initially orthogonal to $\operatorname{grad} H(0)$. Hence, the Lyapunov vectors $\mathbf{V}_{1}(t), \ldots, \mathbf{V}_{N}(t)$ are always orthogonal to $\operatorname{grad} H(t)$, since the $N$-dimensional space spanned by $\mathbf{V}_{1}(t), \ldots, \mathbf{V}_{N}(t)$ is the same as that spanned by $\mathbf{X}_{1}(t), \ldots, \mathbf{X}_{N}(t)$. For the proof of the property (ii), we use the fact that the Hamiltonian vector field $\mathbf{X}_{H}(t)$ is a solution to the linearized Hamilton's Eq. (2), so that one has $\mathbf{X}_{N}(t)=\mathbf{X}_{H}(t)$. Then, $\mathbf{X}_{H}(t)$ is in the $N$-dimensional space spanned by $\mathbf{X}_{1}(t), \ldots, \mathbf{X}_{N}(t)$, and hence, in that spanned by $\mathbf{V}_{1}(t), \ldots, \mathbf{V}_{N}(t)$. By definition, the Lyapunov vectors $\mathbf{V}_{N+1}(t), \ldots, \mathbf{V}_{2 N}(t)$ are orthogonal to $\mathbf{V}_{1}(t), \ldots, \mathbf{V}_{N}(t)$, and hence, to $\mathbf{X}_{H}(t)$.

The above two properties will be confirmed, as well, by numerical calculations for a model system in a later section. Moreover, by numerical calculations in the usual method, we will observe that $\mathbf{V}_{N+2}(t), \ldots, \mathbf{V}_{2 N}(t)$ are not always orthogonal to $\operatorname{grad} H(t)$, and that $\mathbf{V}_{1}(t), \ldots, \mathbf{V}_{N-1}(t)$ are not always orthogonal to $\mathbf{X}_{H}(t)$, either. We recall here that, in the geometric method, Lyapunov vectors $\mathbf{V}_{N+2}^{(g)}(s), \ldots, \mathbf{V}_{2 N}^{(g)}(s)$ are always orthogonal to $\operatorname{grad} K(s)$, and that $\mathbf{V}_{1}^{(g)}(s), \ldots, \mathbf{V}_{N-1}^{(g)}(s)$ are always orthogonal to $\mathbf{X}_{K}(s)$, which will be confirmed, as well, by numerical calculations for the model system. Here, by $\mathbf{V}_{a}^{(g)}$ and $\mathbf{V}_{a}$, we denote the Lyapunov vectors that are obtained in the geometric method and in the usual method, respectively, to tell the difference between them.

\section{Initial conditions}

To compare numerical computation results calculated both in the geometric method and in the usual method, we have to set both Hamilton's equations of motion and linearized equations of motion to share the same initial conditions. Hence, in particular, we come to require that the initial conditions for linearized equations of motion are taken to be subject to the conditions (a) and (b) mentioned in Sec. V B in the usual method as well as in the geometric method. In this section, we discuss how one may set such initial conditions, in spite of the difference between metrics used.

We take a number of initial values, $P(0)$ $=\left(q^{j}(0), p_{j}(0)\right)$, for Hamiltonian flows on $T^{*} M_{J}$ in such a manner that $\operatorname{grad} V$ vanishes at the initial point $P(0)$, where $\operatorname{grad} V$ is defined with respect to both the Euclidean metric and the Jacobi metric on the configuration space $M_{J}$, but the equation $\operatorname{grad} V=0$ defines the same points, independently of the metric chosen. Since the phase spaces in both methods are in common, and since Hamiltonian flows in both methods are also in common up to the change of parameters, we will obtain a number of Hamiltonian flows in common after integration. We also have to note that the condition $\operatorname{grad} V$ $=0$ at the initial point implies that the Christoffel symbols $\Gamma_{j k}^{i}$ 's defined by Eq. (18) vanish also there, so that the Jacobi metric is put, at the initial point, in the form

$$
\left.\tilde{\mathbf{g}}_{J}\right|_{P(0)}=2 W_{0} \delta_{i j} \mathrm{~d} x^{i} \otimes \mathrm{d} x^{j}+\left(2 W_{0}\right)^{-1} \delta^{i j} \mathrm{~d} p_{i} \otimes \mathrm{d} p_{j},
$$

where $W_{0}=E-V(P(0))$.

Let $\mathbf{X}_{a}^{(g)}$ and $\mathbf{X}_{a}$ denote solutions to linearized Eqs. (32) and (2), respectively.

According to the procedure stated in Sec. IV, initial conditions for linearized Eqs. (32) in the geometric method are set as follows:

(a) $\mathbf{X}_{N}^{(g)}(0)=\mathbf{X}_{K}(0), \quad \mathbf{X}_{N+1}^{(g)}(0)=\operatorname{grad} K(0)$,

(b) $\mathbf{X}_{a}^{(g)}(0) \in E_{P(0)} \cap H_{P(0)}, \quad a=1, \ldots, N-1, \quad \mathbf{X}_{b}^{(g)}(0)$ $\in E_{P(0)} \cap V_{P(0)}, \quad b=N+2, \ldots, 2 N$.

See Eqs. (26) and (33) for the definitions of $H_{P(s)}, V_{P(s)}$, and $E_{P(s)}$.

Initial conditions for the linearized Eqs. (2) in the usual method are set as

$$
\mathbf{X}_{a}(0)=\mathbf{X}_{a}^{(g)}(0), \quad a=1, \ldots, 2 N .
$$

We here have to verify that these initial vectors $\mathbf{X}_{a}(0), a$ $=1, \ldots, 2 N$, are indeed subject to the initial conditions (a) and (b) stated in Sec. V B. The verification of this is carried out as follows: By definition, one has $\mathbf{X}_{N}(0)=\mathbf{X}_{K}(0)$, and further $\mathbf{X}_{K}(0)=\mathbf{X}_{H}(0) /[2(E-V[q(0)])]$ from Eq. (37), so that $\mathbf{X}_{N}(0)=\mathbf{X}_{H}(0) /[2(E-V[q(0)])]$. The constant factor $2(E-V[q(0)])$ causes no serious problem, since we are interested in orthogonal relations between initial vectors. Moreover, it is an easy matter to see that $\mathbf{X}_{N+1}(0)$ $=\operatorname{grad} K(0)=\operatorname{grad} H(0)$ on account of the assumption $\operatorname{grad} V(0)=0$ at the initial point, where we note that $\operatorname{grad} K$ and $\operatorname{grad} H$ are taken with respect to metrics, $\widetilde{\mathbf{g}}_{J}$ and $\tilde{\mathbf{g}}_{E}$, respectively. To verify that the other initial vectors, $\mathbf{X}_{a}(0), a=1, \ldots, N-1, N+2, \ldots, 2 N$, are orthogonal to $\mathbf{X}_{H}(0)$ and to $\operatorname{grad} H(0)$, we use the following four facts:

(i) $\mathbf{X}_{1}(0), \ldots, \mathbf{X}_{N-1}(0) \in E_{P(0)} \cap H_{P(0)}$,

(ii) $\mathbf{X}_{N+2}(0), \ldots, \mathbf{X}_{2 N}(0) \in E_{P(0)} \cap V_{P(0)}$,

(iii) $H_{P(0)}$ and $V_{P(0)}$ are orthogonal with respect to the Euclidean metric, as is seen from Eq. (38),

(iv) restricted to the subspaces $H_{P(0)}$ and $V_{P(0)}$, the Jacobi metric and the Euclidean metric are conformal to each other,

$$
\begin{gathered}
\left.\tilde{\mathbf{g}}_{J}\right|_{P(0)}\left(\mathbf{X}_{1}, \mathbf{X}_{2}\right)=\left.2(E-V) \tilde{\mathbf{g}}_{E}\right|_{P(0)}\left(\mathbf{X}_{1}, \mathbf{X}_{2}\right), \\
\mathbf{X}_{1}, \mathbf{X}_{2} \in H_{P(0)}, \\
\left.\tilde{\mathbf{g}}_{J}\right|_{P(0)}\left(\mathbf{X}_{1}, \mathbf{X}_{2}\right)=\left.\tilde{\mathbf{g}}_{E}\right|_{P(0)}\left(\mathbf{X}_{1}, \mathbf{X}_{2}\right) /[2(E-V)], \\
\mathbf{X}_{1}, \mathbf{X}_{2} \in V_{P(0)} .
\end{gathered}
$$

It then turns out from (i) and (iv) that $\mathbf{X}_{1}(0), \ldots, \mathbf{X}_{N-1}(0)$ are also orthogonal to $\mathbf{X}_{N}(0)$ with respect to $\left.\tilde{\mathbf{g}}_{E}\right|_{P(0)}$, and further from (ii) and (iii) that they are also orthogonal to $\mathbf{X}_{N+1}(0)$ with respect to $\left.\tilde{\mathbf{g}}_{E}\right|_{P(0)}$. A similar statement for $\mathbf{X}_{N+2}(0), \ldots, \mathbf{X}_{2 N}(0)$ holds true.

\section{A model system}

The model system we are to consider here is a natural Hamiltonian system with three degrees of freedom that has interactions of Hénon-Heiles type, 


$$
\begin{gathered}
H(q, p)=\sum_{i=1}^{3}\left[\frac{1}{2} p_{i}^{2}+V_{H H}\left(q^{i}, q^{i+1}\right)\right] \\
V_{H H}(x, y)=x^{2} y-\frac{1}{3} y^{3}
\end{gathered}
$$

where $q^{4}=q^{1}$. Hamiltonian vector fields both in the geometric method and in the usual method, denoted by $\mathbf{X}_{K}$ and $\mathbf{X}_{H}$, respectively, are given by Eq. (36) with $g^{i j}=\delta^{i j} /[2(E-V)]$ and $V=\sum_{i=1}^{3} V_{H H}\left(q^{i}, q^{i+1}\right)$.

Hamiltonian flows of $\mathbf{X}_{H}$ for the Hamiltonian (39) are numerically calculated by the use of the fourth-order symplectic integrator [16], which is a numerical integration method on the basis of discrete time evolution with each step an explicit symplectic mapping. Initial conditions for Hamilton's equations of motion are set as $q^{i}(0)=0$ and $p_{i}(0)$ $=\alpha \gamma_{i} \quad(i=1,2,3)$, where $\gamma_{i}$ 's are random values obtained from the uniform distribution function on the interval $[0,1]$, and the constant $\alpha$ is determined so as to satisfy the energy condition $\sum_{i=1}^{3}\left[p_{i}(0)\right]^{2} / 2=E$. For the initial values $q^{i}(0)$ $=0$, we verify easily that the condition $\operatorname{grad} V(0)=0$ is satisfied, which was assumed in the previous Sec. V C. To integrate the linearized Hamilton's equations of motion (2), we take an alternative method, that is, we choose to linearize, along a certain Hamiltonian flow, the sequence of symplectic mappings already obtained on the symplectic integrator algorithm. To our knowledge of explicit symplectic integrators, the symplectic integrator used here and another symplectic algorithm proposed in [17] are set up on the assumption that Hamiltonians are of the form $H(q, p)=T(p)+V(q)$, so that those algorithms are not applicable to the numerical integration of Hamilton's equations of motion with Hamiltonians of the form $K(q, p)=\sum_{i=1}^{3} p_{i}^{2} /[4[E-V(q)]]$. This means that we have to take another algorithm to integrate Hamilton's equations of motion in our geometric method for Lyapunov analysis. What we use in this article is an implicit but symplectic sixth-order Runge-Kutta method (Kuntzmann and Butcher method [18], see Appendix B). However, we have to note here that we do not need to apply that Runge-Kutta method to integrate numerically Hamilton's equations of motion for $K$, since the solutions to Eq. (21) coincide with Hamiltonian flows already obtained by the explicit symplectic integrator up to the parameter change. We apply the implicit Runge-Kutta method to the numerical integration of the lifted Jacobi Eqs. (32), the linearized Hamilton's equations of motion for $K$. The implicit Runge-Kutta method, however, requires an additional process of numerical computation. In fact, we need to calculate the inverse of a $6 N$ $\times 6 N$ matrix at each step of the integration, where $N$ denotes the degrees of freedom. For this reason, the CPU time we have needed to integrate the lifted Jacobi equations by the implicit Runge-Kutta algorithm is about 26 times as long as the CPU time we have needed to integrate the linearized Hamilton's equations of motion for $H$ by the explicit symplectic integrator. We have set the unit time slice as wide as $h=2.5 \times 10^{-6}$ both for the explicit symplectic integrator and the Runge-Kutta algorithm.

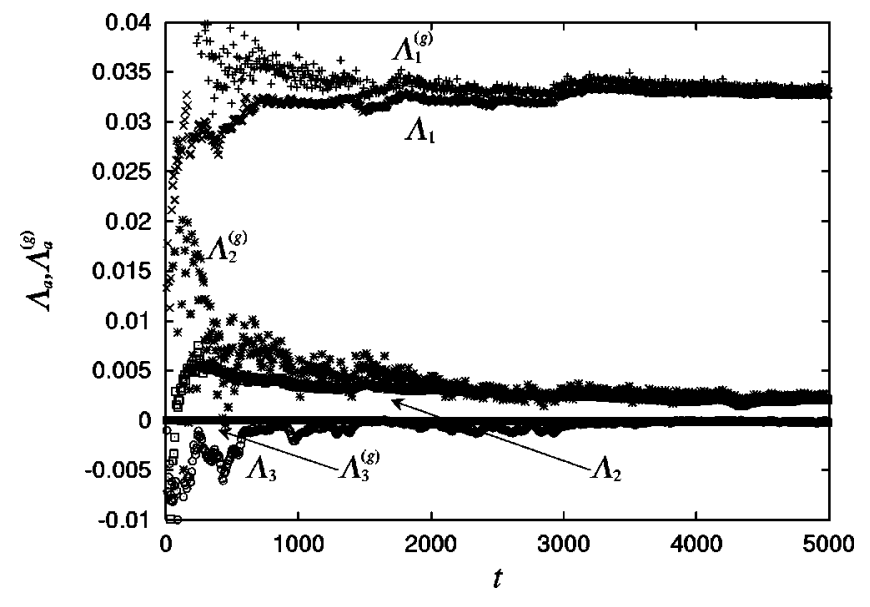

FIG. 1. Convergence of Lyapunov exponents with $E=0.04$. Curves represent graphs of $\Lambda_{a}^{(g)}$ and $\Lambda_{a}(a=1,2,3)$, where $\Lambda_{a}^{(g)}$ and $\Lambda_{a}$, functions in the time parameter, are obtained by the geometric method and by the usual method, respectively.

\section{E. Results of numerical calculations}

Figure 1 shows that Lyapunov exponents calculated in both methods have indeed definite values for $E=0.04$, where $\Lambda_{a}$ 's and $\Lambda_{a}^{(g)}$ 's are defined, respectively, to be

$$
\begin{aligned}
\Lambda_{a}^{(g)}(t) & =\frac{1}{t} \ln \frac{\left\|V_{a}(s(t))\right\|}{\left\|V_{a}(0)\right\|}, \quad \Lambda_{a}(t)=\frac{1}{t} \ln \frac{\left\|V_{a}(t)\right\|}{\left\|V_{a}(0)\right\|}, \\
a & =1,2,3,
\end{aligned}
$$

which are supposed to be convergent to Lyapunov exponents; $\lim _{t \rightarrow \infty} \Lambda_{a}(t)=\lambda_{a}$ and $\lim _{t \rightarrow \infty} \Lambda_{a}^{(g)}(t)=\lambda_{a}^{(g)}$. Here, the quantities with the superscript $(g)$ are those used in the geometric method. However, to compare the numerical results, we have made $\Lambda_{a}^{(g)}(s)$ into a function of $t$ by means of the parameter change. It is to be noted here that $\Lambda_{3}^{(g)}(s)$ always vanishes on account of the fact that $\left\|\mathbf{X}_{3}^{(g)}\right\|=\left\|\mathbf{X}_{K}\right\|=2 K$ $=$ constant. For $E=0.01,0.02$, and 0.03 , we have obtained also definite Lyapunov exponents, which are shown in Fig. 2 along with the dependence on energy. Figure 2 also shows that the Lyapunov exponents, $\lambda_{a}$ and $\lambda_{a}^{(g)}$, calculated in both methods coincide with each other, which means that the Lyapunov exponents are obtained independently of the choice of methods, geometric or usual.

We remark here that if one uses the Jacobi Eqs. (6), a second-order differential equation, to calculate the exponential growth rates of trajectories, one may obtain the same value as that obtained in the usual method. For example, for the Fermi-Pasta-Ulam $\beta$ model, the largest Lyapunov exponent is calculated by using a $2 N$-dimensional vector $\left(X^{i}, \mathrm{~d} X^{i} / \mathrm{d} t\right)[19]$, where $\left[X^{i}(t)\right]$ is a solution to the Jacobi Eqs. (6) and the Euclidean metric is used for the $2 N$-dimensional vector. According to [19], the resultant value of the exponent coincides with the largest Lyapunov exponent obtained in the usual method. This might suggest that to calculate the largest Lyapunov exponent, one does not need to work with the cotangent spaces. However, the advantage of the geometric method developed in this article is that after the geometric method, we may obtain all the 


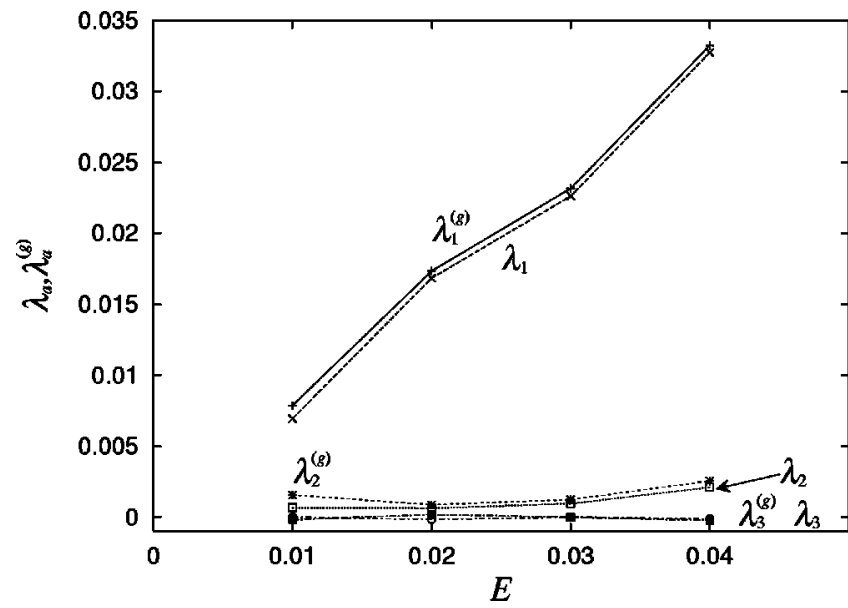

FIG. 2. Comparison of Lyapunov exponents obtained by both the geometric method and the usual method. By $\lambda_{a}^{(g)}$ and $\lambda_{a}$, we denote Lyapunov exponents obtained by the geometric method and by the usual method, respectively. Numerical results obtained in both methods are in good agreement.

Lyapunov exponents along with the Lyapunov vectors among which two vectors associated with the vanishing Lyapunov exponents may be separated out from the others. This may be observed in Figs. 3 and 4.

From Figs. 3 and 4, we will observe that the Lyapunov vectors calculated numerically in the geometric method satisfy the requirements stated in Sec. I and that the Lyapunov vectors calculated in the usual method have the property shown in Sec. V B.
Figure 3 provides temporal evolutions of inner products between normalized Lyapunov vectors and the normalized tangent vector to a Hamiltonian flow. The inner products both in the geometric method and in the usual method are denoted by $t_{a}^{(g)}$ and by $t_{a}$, respectively,

$$
t_{a}^{(g)}=\tilde{\mathbf{g}}_{J}\left(\frac{\mathbf{V}_{a}^{(g)}}{\left\|\mathbf{V}_{a}^{(g)}\right\|}, \mathbf{X}_{K}\right), \quad t_{a}=\tilde{\mathbf{g}}_{E}\left(\frac{\mathbf{V}_{a}}{\left\|\mathbf{V}_{a}\right\|}, \frac{\mathbf{X}_{H}}{\left\|\mathbf{X}_{H}\right\|}\right)
$$

Figure 3 shows that all the Lyapunov vectors except for $\mathbf{V}_{3}^{(g)}$ in the geometric method are orthogonal to $\mathbf{X}_{K}$, and that the normalized $\mathbf{V}_{3}^{(g)}$ is equal to $\mathbf{X}_{K}$. On the other hand, we observe also from Fig. 3 that $\mathbf{V}_{1}$ and $\mathbf{V}_{2}$ in the usual method are not always orthogonal to $\mathbf{X}_{H}$, and that the normalized $\mathbf{V}_{3}$ does not equal $\mathbf{X}_{H} /\left\|\mathbf{X}_{H}\right\|$, either. In particular, we remark that $t_{2}$ takes values around unity in opposition to our requirement.

Figure 4 provides temporal evolutions of inner products between normalized Lyapunov vectors and the normalized gradient vector of the Hamiltonian, and the inner product both in the geometric method and in the usual method is denoted by the symbol $n_{a}^{(g)}$ and $n_{a}$, respectively,

$$
n_{a}^{(g)}=\tilde{\mathbf{g}}_{J}\left(\frac{\mathbf{V}_{a}^{(g)}}{\left\|\mathbf{V}_{a}^{(g)}\right\|}, \operatorname{grad} K\right), \quad n_{a}=\tilde{\mathbf{g}}_{E}\left(\frac{\mathbf{V}_{a}}{\left\|\mathbf{V}_{a}\right\|}, \frac{\operatorname{grad} H}{\|\operatorname{grad} H\|}\right)
$$

All the Lyapunov vectors except for $\mathbf{V}_{4}^{(g)}$ are observed to be orthogonal to $\operatorname{grad} K$, and $\mathbf{V}_{4}^{(g)}$ to be collinear to $\operatorname{grad} K$ in the geometric method, as is expected. On the other hand, the
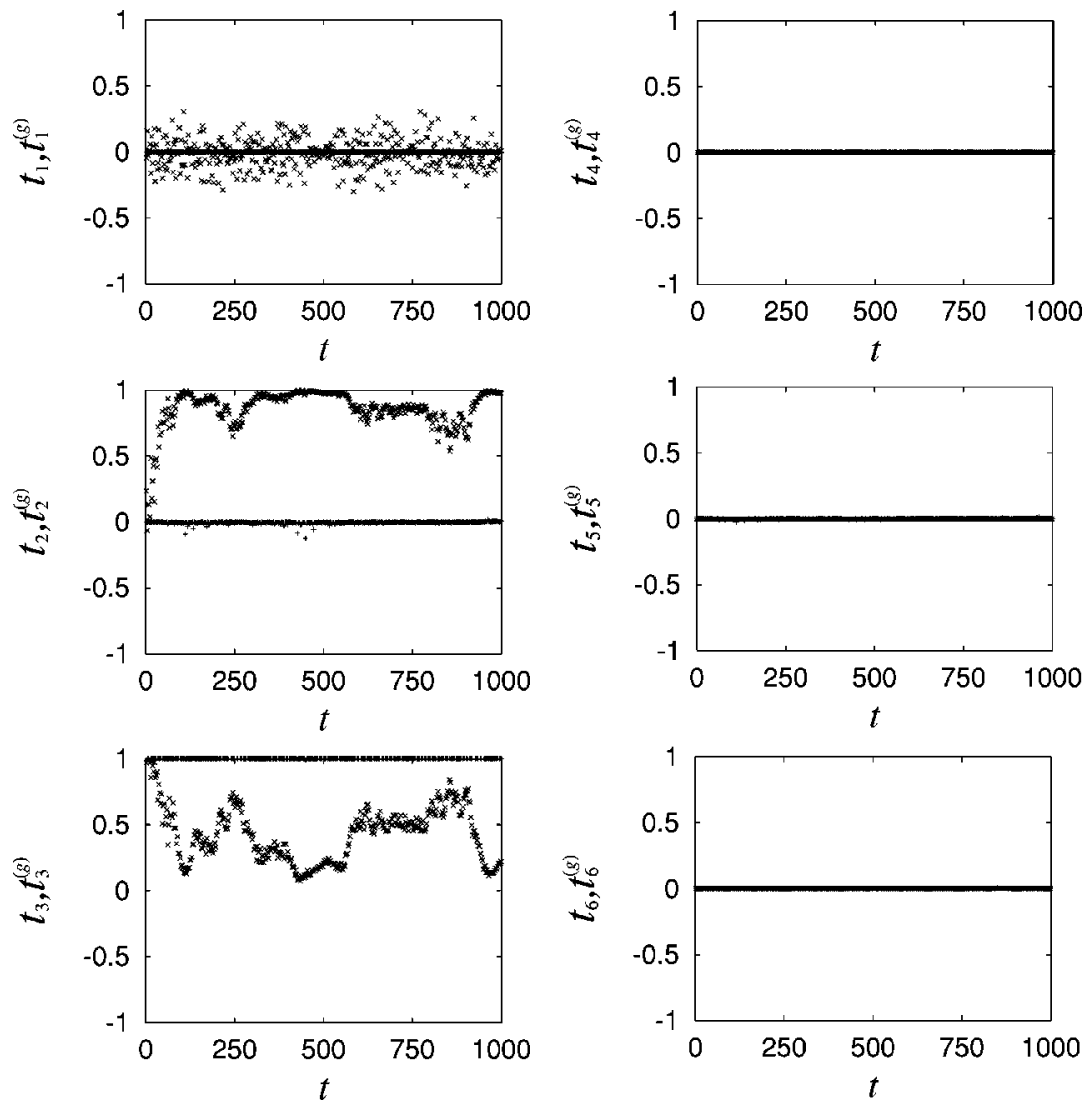

FIG. 3. Temporal evolutions of inner products between the normalized tangent vector of a Hamiltonian flow and normalized Lyapunov vectors. The energy is set at $E=0.04$. In (a), (b), and (c), straight lines are graphs of $t_{a}^{(g)}$ against the time parameter in the geometric method, and broken curves are from the usual method, providing the graphs of $t_{a}$. The first and second Lyapunov vectors $\mathbf{V}_{1}^{(g)}, \mathbf{V}_{2}^{(g)}$ are always orthogonal to the tangent direction to a Hamiltonian flow $\mathbf{X}_{K}$ in the geometric method, but $\mathbf{V}_{1}, \mathbf{V}_{2}$ are not always orthogonal to $\mathbf{X}_{H}$ in the usual method. Moreover, the third Lyapunov vector always points to the direction of $\mathbf{X}_{K}$ in the geometric method, but does not point to the direction of $\mathbf{X}_{H}$ in the usual method. In (d), (e), and (f), only straight lines are drawn, which are graphs from both methods, but they coincide with each other. 

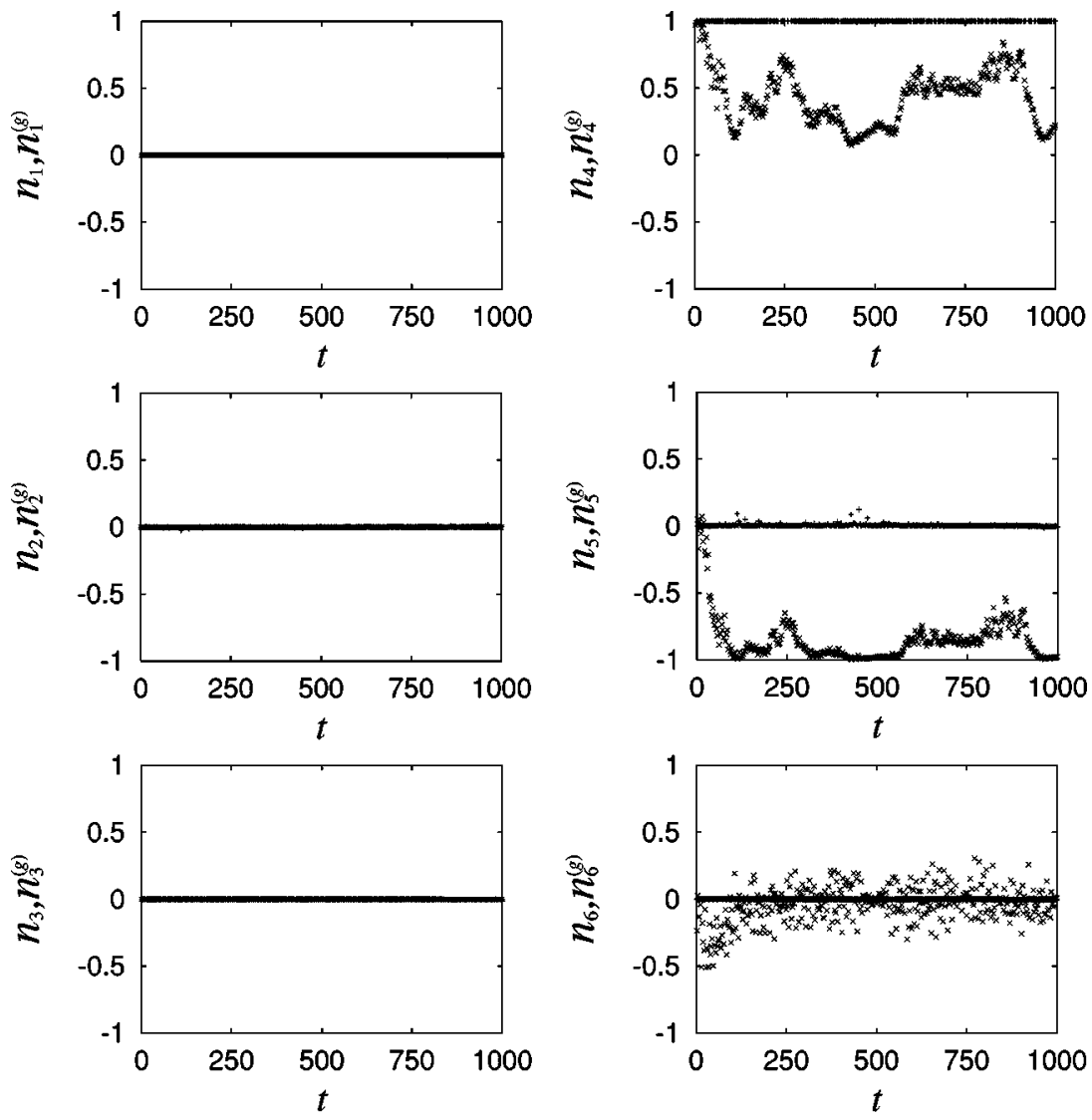

FIG. 4. Temporal evolutions of inner products between the normalized gradient vector of the Hamiltonian function and the normalized Lyapunov vectors. The energy is set at $E=0.04$. In (d), (e), and (f), straight lines are graphs of $n_{a}^{(g)}$ against the time parameter in the geometric method, and broken curves are from the usual method, providing the graph of $n_{a}$. The 4th Lyapunov vector $\mathbf{V}_{4}^{(g)}$ always points to the gradient direction of the Hamiltonian function $K$, but $\mathbf{V}_{4}$ does not always point to $\operatorname{grad} H$ in the usual method. Moreover, the 5 th and 6th Lyapunov vectors $\mathbf{V}_{5}^{(g)}, \mathbf{V}_{6}^{(g)}$ are always orthogonal to $\operatorname{grad} K$ in the geometric method, and $\mathbf{V}_{5}, \mathbf{V}_{6}$ are not so to $\operatorname{grad} H$ in the usual method. In (a), (b), and (c), straight lines from the two methods are drawn, but each of them looks like a single line because of coincidence.
Lyapunov vectors $\mathbf{V}_{5}, \mathbf{V}_{6}$ in the usual method are not always orthogonal to $\operatorname{grad} H$, and $\mathbf{V}_{4}$ does not point to the direction of $\operatorname{grad} H$, either. In particular, $n_{5}$ in the usual method is far from vanishing, taking values around minus unity.

These observations agree to what we expect from the theory described in Secs. IV B and V B. We note in conclusion that tiny fluctuations around straight lines in Figs. 3 and 4, in particular, Figs. 3(b) and 4(e), seem to be numerical errors due to the factor $1 /[2(E-V)]$ included in the metric $g^{i j}$, Christoffel symbol $\Gamma_{j k}^{i}$, and the Riemann curvature tensor $R_{i j k l}$.

\section{CONCLUDING REMARKS}

In this paper, we have developed a geometric method in Lyapunov analysis for natural Hamiltonian systems with $N$ degrees of freedom, which is set up on the cotangent bundle of a Riemannian manifold endowed with the Jacobi metric. In contrast with our geometric method, the old or alreadyknown geometric method is established on the Riemannian manifold with the Jacobi metric. According to that method, one brings Newton's equations of motion for a natural dynamical system into geodesic equations for the Jacobi metric and uses Jacobi equations, linearized geodesic equations, to analyze orbital instability of trajectories. However, the Jacobi equations are second-order differential equations, while Lyapunov exponents and vectors are defined through firstorder differential equations. We then need a first-order differential equation to apply Lyapunov analysis. According to our method, the Jacobi equations are lifted from Riemannian manifolds to their cotangent bundles to take the form of firstorder differential equations.

When the geometric method is applied, a question arises as to whether Lyapunov exponents remain unchanged in their values or not, in comparison with those obtained in the usual method. As we have already pointed out, the linearized equations in both methods are different from each other and cannot be transformed to each other through the parameter transformation $\mathrm{d} s=2[E-V(q)] \mathrm{d} t$, while the equations of motion in both methods are transformed to each other through the same parameter transformation. However, the numerical computation has shown that the values of Lyapunov exponents coincide with each other, independently of the choice of methods applied, as far as the model system with three degrees of freedom is taken. We guess that the Lyapunov exponents are long-term averaged values, so that they are independent of the choice of Lyapunov vectors along trajectories, while Lyapunov vectors depend on the choice of methods. As for the parameters of trajectories in both methods, we assume that the change of parameters must be subject to the condition $0<\mathrm{d} s / \mathrm{d} t<\infty$ along trajectories. On this account, we expect that Lyapunov exponents are independent of the choice of methods for calculation. We will find indeed the coincidence of Lyapunov exponents in both methods from numerical computations for other model systems. Further, observations made from the Lyapunov exponents are expected to be independent of the choice of methods. For instance, a characteristics of the graph of Lyapunov spectra $\lambda_{i}$ against $i / N, i=1, \ldots, N \quad[20,21]$, which are observed in the usual method for a wide class of 
Hamiltonian systems having nearest-neighbor interactions, will be found, in the geometric method as well, to be the same as that observed already in the usual method. We wish our geometric method may afford a fresh insight into the observation through Lyapunov vectors.

In our geometric method developed in this paper, we may choose Lyapunov vectors so as to satisfy the following requirements: (i) Lyapunov vectors except for $N$ th and $(N$ $+1)$-th vectors are always orthogonal to both the tangent direction to a trajectory and the gradient direction of the Hamiltonian function, (ii) $N$ th Lyapunov vector points to the tangent direction of the trajectory, $\mathbf{X}_{K}$, and (iii) $(N+1)$-th Lyapunov vector points to the gradient direction of the Hamiltonian function, $\operatorname{grad} K$. Along with such Lyapunov vectors, we may analyze orbital instability of Hamiltonian flows in phase spaces without influence of the two marginal directions pointed by $\mathbf{X}_{K}$ and $\operatorname{grad} K$ that have vanishing Lyapunov exponents, $\lambda_{N}=\lambda_{N+1}=0$. Moreover, the $N$ th and the $(N+1)$-th local Lyapunov exponents, which are averages of exponential growth rate in finite time, vanish on any time interval. The local Lyapunov exponents in the usual method are used, for instance, to distinguish nearly integrable systems from the others [22].

In this paper, we have considered the Hamiltonian function of the form $H(q, p)=\frac{1}{2} \sum_{i j} \delta^{i j} p_{i} p_{j}+V(q)$ and developed the geometric method in Lyapunov analysis. However, the geometric method may be established for Hamiltonian functions of the form $H(q, p)=\frac{1}{2} \sum_{i j} a^{i j}(q) p_{i} p_{j}+V(q)$, where $\left[a^{i j}(q)\right]$ is the inverse of a metric tensor $\left[a_{i j}(q)\right]$. In this case, the Jacobi metric is defined to be $g_{i j}(q)=2[E$ $-V(q)] a_{i j}(q)$, and geodesic equations for this metric

$$
\frac{\mathrm{d}^{2} q^{i}}{\mathrm{~d} s^{2}}+\Gamma_{j k}^{i} \frac{\mathrm{d} q^{j}}{\mathrm{~d} s} \frac{\mathrm{d} q^{k}}{\mathrm{~d} s}=0
$$

prove to be equivalent to Newton's equations of motion

$$
\frac{\mathrm{d}^{2} q^{i}}{\mathrm{~d} t^{2}}+\left\{\begin{array}{c}
i \\
j k
\end{array}\right\} \frac{\mathrm{d} q^{j}}{\mathrm{~d} t} \frac{\mathrm{d} q^{k}}{\mathrm{~d} t}=-a^{i j} \frac{\partial V}{\partial q^{j}},
$$

with the total energy fixed at $E$, where $\Gamma_{j k}^{i}$ and $\left\{\begin{array}{c}i \\ j k\end{array}\right\}$ are the Christoffel symbols formed from the metric $g_{i j}$ and $a_{i j}$, respectively, and $s$ is the length parameter for the Jacobi metric $g_{i j}$, which is related to the parameter $t$ by $\mathrm{d} s / \mathrm{d} t=2[E$ $-V(q)]$. The geometric method we have developed in the Lyapunov analysis of linearized Hamilton's equations of motion on the cotangent bundle is independent of the choice of the Riemannian metric chosen, so that the theorem stated in Sec. IV B holds also true in this case. Hence, we may find Lyapunov vectors that satisfy the requirements mentioned frequently.

\section{ACKNOWLEDGMENTS}

The authors would like to thank Dr. Y. Uwano for valuable discussions. This work is supported by the Grant-In-Aid for Scientific Research of the Ministry of Education, Culture,
Sports, Science and Technology of Japan (12750060 and 11640199).

\section{APPENDIX A: GEOMETRY OF COTANGENT BUNDLES}

Vector fields and Levi-Civita connection on a Riemannian manifold $M$ are lifted to the cotangent bundle $T^{*} M$, and thereby the relation between geodesics on $M$ and geodesic flows on $T^{*} M$ will be made clear in geometric fashion.

\section{Lift of vector fields on $M$}

The cotangent bundle $T^{*} M$ is endowed with the standard one-form $\theta$, which is expressed locally as $\theta=p_{i} d x^{i}$. Note that the $\theta$ is defined globally on $T^{*} M$. This may be seen from the coordinate transformation on the nonempty intersection (19). The exterior derivative of $\theta, \omega:=\mathrm{d} \theta$, is the standard symplectic form on $T^{*} M$.

For vector fields on $M$, a way to lift them is not unique. A canonical way is given as follows: For $\mathbf{Y} \in \bar{X}(M)$, the lifted vector field $\widetilde{\mathbf{Y}}$ is defined through the conditions

$$
\pi_{*} \tilde{\mathbf{Y}}=\mathbf{Y}, \quad \mathcal{L}_{\tilde{\mathbf{Y}}} \theta=0
$$

where $\pi_{*}$ is the differential of the canonical projection $\pi$, and $\mathcal{L}$ denotes the Lie derivation. For $\mathbf{Y}=Y^{i} \partial_{i}$, a straightforward calculation shows that the $\tilde{\mathbf{Y}}$ is put in the form

$$
\widetilde{\mathbf{Y}}=Y^{i} \frac{\partial}{\partial x^{i}}-p_{j} \frac{\partial Y^{j}}{\partial x^{i}} \frac{\partial}{\partial p_{i}} .
$$

Furthermore, owing to Cartan's formula, $\mathcal{L}_{\tilde{\mathbf{Y}}} \theta=\mathrm{d}[\iota(\widetilde{\mathbf{Y}}) \theta]$ $+\iota(\widetilde{\mathbf{Y}}) \mathrm{d} \theta$, along with $\iota(\widetilde{\mathbf{Y}}) \theta=\theta(\widetilde{\mathbf{Y}})$, the latter of the conditions (A1) implies that $-d[\theta(\tilde{\mathbf{Y}})]=\iota(\tilde{\mathbf{Y}}) \omega$, which then shows that the $\widetilde{\mathbf{Y}}$ becomes the Hamiltonian vector field associated with $F:=\theta(\widetilde{\mathbf{Y}})=p_{i} Y^{i}$. Thus, one has

$$
\tilde{\mathbf{Y}}=\mathbf{X}_{F}=\frac{\partial F}{\partial p_{i}} \frac{\partial}{\partial x^{i}}-\frac{\partial F}{\partial x_{i}} \frac{\partial}{\partial p^{i}} .
$$

With respect to the adapted frame, the canonical lift $\tilde{\mathbf{Y}}$ takes the form

$$
\widetilde{\mathbf{Y}}=Y^{i} D_{i}-p_{j} \nabla_{i} Y^{j} D_{\bar{i}}
$$

where

$$
\nabla_{i} Y^{j}=\frac{\partial Y^{j}}{\partial x^{i}}+\Gamma_{i k}^{j} Y^{k}
$$

In addition to the canonical lift, one may define another lift; for a vector field $\mathbf{Y}=Y^{i} \partial_{i}$ on $M$, the horizontal lift of $\mathbf{Y}$ is given on $T^{*} M$ by

$$
\widetilde{\mathbf{Y}}^{h}=Y^{i} D_{i}
$$


From the transformation rule (25), the horizontal lift is shown to be defined independently of the choice of adapted frames.

A curve $x(t)$ in $M$ is also lifted horizontally; a curve $\tilde{x}^{h}(t)$ in $T^{*} M$ is called a horizontal lift of $x(t)$, if $\pi\left[\tilde{x}^{h}(t)\right]$ $=x(t)$ and if the tangent vector to $\tilde{x}^{h}(t)$ is horizontal. To give an example of horizontal lifts of curves, we consider a geodesic $x(s)$ with $s$ the arc length parameter. Let $\boldsymbol{\xi}(s)$ denote its tangent vector and let $p_{i}(s)=g_{i j} \xi^{j}(s)$. Then, a curve $[x(s), p(s)]$ in the cotangent bundle $T^{*} M$ is shown to be a horizontal curve. In fact, differentiation of $[x(s), p(s)]$ with respect to $s$ along with the geodesic equation for $x(s)$ provides

$$
\frac{\mathrm{d}}{\mathrm{d} s}\left[x^{i}(s), p_{i}(s)\right]=\left(\xi^{i}, \Gamma_{i j}^{k} p_{k} \xi^{j}\right)=\xi^{i}(s) D_{i},
$$

as is wanted. From Eq. (A6) along with $\xi^{i}(s)=g^{i \ell} p /(s)$, we observe that the curve $[x(s), p(s)]$ is a geodesic flow, an integral curve of $\mathbf{X}_{K}$ [see Eq. (27)].

Now we assume that $\boldsymbol{\xi}$ is a tangent vector field to a congruence of geodesics in $M$. According to Eq. (A5), we can define the horizontal lift $\widetilde{\boldsymbol{\xi}}^{h}$ on $T^{*} M$. With the restriction $p_{i}=g_{i j} \xi^{j}(x)$ imposed, the Hamiltonian vector field $\mathbf{X}_{K}$ becomes equal to the horizontal lift $\widetilde{\xi}^{h}$. Hence, a congruence of geodesics in $M$ is lifted to a family of geodesic flows in $T^{*} M$ along with $\widetilde{\xi}^{h}=\mathbf{X}_{K}$.

We proceed to discuss lifts of geodesic deviations. Let $\mathbf{Y}(s)$ be a vector field defined along the geodesic $x(s)$. We define a vector field $\mathbf{X}(s)$ along a geodesic flow $[x(s), p(s)]$ with $p_{i}(s)=g_{i j} \xi^{j}(s)$, by

$$
\mathbf{X}=Y^{i} D_{i}+g_{i j}\left(\nabla_{\xi} \mathbf{Y}\right)^{j} D_{\bar{i}}
$$

We note here that the $\mathbf{X}(s)$ is defined independently of the choice of adapted frames. If $\mathbf{X}(s)$ satisfies the lifted Jacobi Eq. (32), then the $\mathbf{Y}(s)$ should be a Jacobi field. Conversely, for a given Jacobi field $\mathbf{Y}(s)$ defined along a geodesic $x(s)$, we may form a lifted vector field $\mathbf{X}(s)$ according to Eq. (A7), which is defined along a geodesic flow $P(s)$ $=[x(s), p(s)]$ with $p_{i}(s)=g_{i j} \xi^{j}(s)$. Then, $\mathbf{X}(s)$ solves Eq. (32).

\section{Killing vector fields}

We now wish to investigate the relation between the canonical lift (A3) and the lift (A7), where $\boldsymbol{\xi}$ is viewed as the tangent vector field to a congruence of geodesics in $M$. To this end, we first consider symmetry of our Hamiltonian system with the Hamiltonian function $K$. We assume here that for a vector field $\mathbf{Y}$ on $M$ the function $F=\theta(\mathbf{Y})=Y^{i} p_{i}$ is a constant of motion; $\mathbf{X}_{K}(F)=-\{K, F\}=0$, where $\{\cdot, \cdot\}$ denotes Poisson bracket. Then one obtains $\left[\mathbf{X}_{K}, \mathbf{X}_{F}\right]$ $=-\mathbf{X}_{\{K, F\}}=0$. This implies that $\tilde{\mathbf{Y}}=\mathbf{X}_{F}$ satisfies the linearized Eq. (32) along any geodesic flow. On the other hand, the condition $\mathbf{X}_{K}(F)=0$ holds, if and only if $\mathbf{Y}$ is a Killing vector field, an infinitesimal isometry, i.e., $\mathcal{L}_{\mathbf{Y}} \mathbf{g}=0$, as is easily seen. It is well known that every Killing vector field satisfies the Jacobi equation along any geodesic.

Now, we assume further that we are given the tangent vector field $\boldsymbol{\xi}$ to a congruence of geodesics in $M$. If restricted on a subspace $L$ determined by $p_{i}=g_{i j} \xi^{j}$ in $T^{*} M$, the canonical lift $\widetilde{\mathbf{Y}}$ of a Killing vector field $\mathbf{Y}$ is expressed as

$$
\left.\tilde{\mathbf{Y}}\right|_{L}=Y^{i} D_{i}-g_{j k} \xi^{k} \nabla_{i} Y^{j} D_{\bar{i}}^{-}=Y^{i} D_{i}+g_{i j}\left(\nabla_{\xi} \mathbf{Y}\right)^{j} D_{\bar{i}}
$$

where use has been made of the formula that

$$
g^{i j} \nabla_{i} Y^{k}+g^{i k} \nabla_{i} Y^{j}=0,
$$

which is a necessary and sufficient condition for $\mathbf{Y}$ to be a Killing vector field. Thus, we have found that if $\mathbf{Y}$ is a Killing vector on $M$, and if the canonical lift $\widetilde{\mathbf{Y}}$ is restricted to $L$ determined by $p_{i}=g_{i j} \xi^{j}$, then $\tilde{\mathbf{Y}} \mid L$ is equal to the lift (A7) with $\boldsymbol{\xi}$ the tangent vector field to a congruence of geodesics.

\section{Levi-Civita connection of $T^{*} M$}

The Levi-Civita connection $\widetilde{\nabla}$ is defined on the cotangent bundle $T^{*} M$ through the Sasaki metric $\widetilde{\mathbf{g}}$. We denote the Christoffel symbols for this connection by $\hat{\Gamma}_{B C}^{A}$;

$$
\widetilde{\nabla}_{\partial_{B}} \partial_{C}=\hat{\Gamma}_{B C}^{A} \partial_{A},
$$

where Roman capital indices run from 1 to $2 m$ and $\partial_{A}$ are the standard frame;

$$
\partial_{i}=\frac{\partial}{\partial x^{i}}, \quad \partial_{\bar{i}}=\frac{\partial}{\partial p_{i}}, \quad i=1, \ldots, m
$$

The Christoffel symbols are given, as usual, by

$$
\hat{\Gamma}_{B C}^{A}=\frac{1}{2} \hat{g}^{A D}\left(\partial_{B} \hat{g}_{C D}+\partial_{C} \hat{g}_{D B}-\partial_{D} \hat{g}_{B C}\right)
$$

where $\hat{g}_{A B}$ are components of $\tilde{\mathbf{g}} ; \hat{g}_{A B}=\tilde{\mathbf{g}}\left(\partial_{A}, \partial_{B}\right)$. We denote the coefficients of the connection $\widetilde{\nabla}$ with respect to the adapted frame by $\widetilde{\Gamma}_{\beta \gamma}^{\alpha}$,

$$
\widetilde{\nabla}_{D_{\beta}} D_{\gamma}=\widetilde{\Gamma}_{\beta \gamma}^{\alpha} D_{\alpha}
$$

where Greek indices also run from 1 to $2 \mathrm{~m}$, but indicating that they are indices for the adapted frame.

Let the functions $\Omega_{\beta \gamma}{ }^{\alpha}$ be defined by

$$
\left[D_{\beta}, D_{\gamma}\right]=\Omega_{\beta \gamma}{ }^{\alpha} D_{\alpha} \text {. }
$$

Then, the torsion-free condition for $\tilde{\nabla}$ is put in the form

$$
\widetilde{\Gamma}_{\beta \gamma}^{\alpha}-\widetilde{\Gamma}_{\gamma \beta}^{\alpha}=\Omega_{\beta \gamma}{ }^{\alpha}
$$

A straightforward calculation yields $\Omega_{\beta \gamma}{ }^{\alpha}$ as follows:

$$
\left[D_{i}, D_{j}\right]=p_{\ell} R_{i j k} D_{\bar{k}}, \quad\left[D_{i}, D_{\bar{j}}\right]=-\Gamma_{i k}^{j} D_{\bar{k}},
$$


TABLE II. Kuntzmann \& Butcher method, order six. The upper-right block is the matrix $\left(a_{i j}\right)$, the left column is the vector $\left(b_{i}\right)$, and the lower raw is the vector $\left(c_{i}\right)$.

\begin{tabular}{l|ccc}
\hline \hline$\frac{1}{2}-\frac{\sqrt{15}}{10}$ & $\frac{5}{36}$ & $\frac{2}{9}-\frac{\sqrt{15}}{15}$ & $\frac{5}{36}-\frac{\sqrt{15}}{30}$ \\
$\frac{1}{2}$ & $\frac{5}{36}+\frac{\sqrt{15}}{24}$ & $\frac{2}{9}$ & $\frac{5}{36}-\frac{\sqrt{15}}{24}$ \\
$\frac{1}{2}+\frac{\sqrt{15}}{10}$ & $\frac{5}{36}+\frac{\sqrt{15}}{30}$ & $\frac{2}{9}+\frac{\sqrt{15}}{15}$ & $\frac{5}{36}$ \\
\hline \hline & $\frac{5}{18}$ & $\frac{4}{9}$ & $\frac{5}{18}$ \\
\hline \hline$\left[D_{\bar{i}}, D_{j}\right]=\Gamma_{j k}^{i} D_{\bar{k}}$, & {$\left[D_{\bar{i}}, D_{\bar{j}}\right]=0$.} & $\widetilde{\nabla}_{\mathbf{X}_{1}} \mathbf{X}_{2}=\left[X_{1}^{\beta} D_{\beta} X_{2}^{\alpha}+\widetilde{\Gamma}_{\beta \gamma}^{\alpha} X_{1}^{\beta} X_{2}^{\gamma}\right] D_{\alpha}$,
\end{tabular}

We are to write out $\widetilde{\Gamma}_{\beta \gamma}^{\alpha}$ in terms of $\tilde{g}_{\alpha \beta}$ and $\Omega_{\beta \gamma}{ }^{\alpha}$, where $\tilde{g}_{\alpha \beta}=\tilde{\mathbf{g}}\left(D_{\alpha}, D_{\beta}\right)$, the components of $\tilde{\mathbf{g}}$ with respect to the adapted frame. The covariant derivative of the metric $\tilde{\mathbf{g}}$ must vanish for all vector fields $\mathbf{X}$ on $T^{*} M ; \widetilde{\nabla}_{\mathbf{X}} \tilde{\mathbf{g}}=0$, so that one has

$$
D_{\beta} \tilde{g}_{\gamma \delta}-\widetilde{\Gamma}_{\beta \gamma}^{\varepsilon} \tilde{g}_{\varepsilon \delta}-\Gamma_{\beta \delta}^{\varepsilon} \tilde{g}_{\gamma \varepsilon}=0 .
$$

Further calculation provides

$$
\begin{aligned}
& D_{\beta} \tilde{g}_{\gamma \delta}+D_{\gamma} \tilde{g}_{\delta \beta}-D_{\delta} \tilde{g}_{\beta \gamma} \\
& \quad=\left(\widetilde{\Gamma}_{\beta \gamma}^{\epsilon}+\widetilde{\Gamma}_{\gamma \beta}^{\epsilon}\right) \tilde{g}_{\epsilon \delta}+\left(\widetilde{\Gamma}_{\beta \delta}^{\epsilon}-\widetilde{\Gamma}_{\delta \beta}^{\epsilon}\right) \tilde{g}_{\epsilon \gamma}+\left(\widetilde{\Gamma}_{\gamma \delta}^{\epsilon}-\widetilde{\Gamma}_{\delta \gamma}^{\epsilon}\right) \tilde{g}_{\epsilon \beta} \\
& \quad=\left(2 \widetilde{\Gamma}_{\beta \gamma}^{\epsilon}-\Omega_{\beta \gamma}{ }^{\epsilon}\right) \tilde{g}_{\epsilon \delta}+\Omega_{\beta \delta} \tilde{g}_{\epsilon \gamma}+\Omega_{\gamma \delta} \tilde{g}_{\epsilon \beta},
\end{aligned}
$$

which results in

$$
\begin{aligned}
\widetilde{\Gamma}_{\beta \gamma}^{\alpha}= & \frac{1}{2} \tilde{g}^{\alpha \delta}\left(D_{\beta} \tilde{g}_{\gamma \delta}+D_{\gamma} \tilde{g}_{\delta \beta}-D_{\delta} \tilde{g}_{\beta \gamma}\right) \\
& +\frac{1}{2}\left(\Omega_{\beta \gamma}{ }^{\alpha}+\Omega^{\alpha}{ }_{\beta \gamma}+\Omega^{\alpha}{ }_{\gamma \beta}\right),
\end{aligned}
$$

where

$$
\Omega_{\beta \gamma}^{\alpha}=\tilde{g}^{\alpha \delta} \Omega_{\delta \beta} \epsilon_{\tilde{g}_{\epsilon \gamma}} .
$$

A straightforward calculation shows that the components $\widetilde{\Gamma}_{\beta \gamma}^{\alpha}$ are given by

$$
\begin{gathered}
\widetilde{\Gamma}_{j k}^{i}=\Gamma_{j k}^{i}, \quad \widetilde{\Gamma}_{j \bar{k}}^{i}=-\frac{1}{2} R_{j}{ }^{i k \ell} p_{\ell}, \quad \widetilde{\Gamma}_{\bar{j} k}^{i}=-\frac{1}{2} R_{k}{ }^{i j \ell} p_{\ell}, \\
\widetilde{\Gamma}_{\bar{j} \bar{k}}^{i}=0, \quad \widetilde{\Gamma}_{j k}^{\bar{i}}=\frac{1}{2} R_{j k i} p_{\ell}, \quad \widetilde{\Gamma}_{j \bar{k}}^{\bar{i}}=-\Gamma_{i j}^{k}, \\
\widetilde{\Gamma}_{\bar{j} k}^{\bar{i}}=0, \quad \widetilde{\Gamma}_{\bar{j} \bar{i}}^{\bar{i}}=0 .
\end{gathered}
$$

Covariant derivatives of vector fields are then expressed, in terms of these coefficients, as where $\left(X_{1}^{\alpha}\right)$ and $\left(X_{2}^{\alpha}\right)$ are components of $\mathbf{X}_{1}$ and $\mathbf{X}_{2}$ with respect to $D_{\alpha}$, respectively. In particular, the covariant derivative of $\mathbf{X}=X^{i} D_{i}+X^{\bar{i}} D_{\bar{i}}^{-}$with respect to the horizontal lift $\widetilde{\boldsymbol{\xi}}^{h}=\xi^{i}(s) D_{i}$ along a geodesic flow as a horizontal lift of a geodesic takes the form

$$
\begin{aligned}
& \left(\widetilde{\nabla}_{\widetilde{\xi}_{h}} \mathbf{X}\right)^{i}=\frac{\mathrm{d} X^{i}}{\mathrm{~d} s}+\Gamma_{k j}^{i} \xi^{k} X^{j}-\frac{1}{2} R_{k}^{i j \ell} p / \xi^{k} X^{\bar{j}}, \\
& \left(\widetilde{\nabla}_{\tilde{\xi} h} \mathbf{X}\right)^{\bar{i}}=\frac{\mathrm{d} X^{\bar{i}}}{\mathrm{~d} s}-\Gamma_{i k}^{j} \xi^{k} X^{\bar{j}}+\frac{1}{2} R_{k j i} \ell p / \xi^{k} X^{j} .
\end{aligned}
$$

If $\mathbf{X}=\widetilde{\boldsymbol{\xi}}^{h}$, these equations give rise to

$$
\tilde{\nabla}_{\widetilde{\boldsymbol{\xi}}^{h}} \widetilde{\boldsymbol{\xi}}^{h}=0
$$

which implies that the horizontal lift $[x(s), p(s)]$ of a geodesic $x(s)$ on $M$ is also a geodesic on $T^{*} M$ with respect to the lifted metric $\tilde{\mathbf{g}}$. We note here that the arc length parameter $\sigma$ with respect to $\tilde{\mathbf{g}}$ reduces to the arc length parameter $s$, if the curve is horizontal.

\section{APPENDIX B: SYMPLECTIC IMPLICIT RUNGE-KUTTA METHOD}

Suppose we are given a dynamical system in $\mathbf{R}^{\prime}$

$$
\frac{\mathrm{d} x}{\mathrm{~d} t}(t)=f(x, t) .
$$

Numerical integration of this equation is performed through discretizing it with time slice $h$. The $s$-stage Runge-Kutta method for integration is given by

$$
x^{\prime}=x+h \sum_{i=1}^{s} b_{i} k_{i}
$$




$$
k_{i}=f\left(x+h \sum_{j=1}^{s} a_{i j} k_{j}, t+c_{i} h\right), \quad i=1, \ldots, s,
$$

where $(x, t)$ goes to $\left(x^{\prime}, t+h\right)$ after one step, and $a_{i j}, b_{i}$, and $c_{i}$ are real constants with $\sum_{i=1}^{s} c_{i}=1$. Note that the second of the above equations defines implicitly $k_{i}$. The threestage Runge-Kutta method, namely the sixth-order Kuntzmann and Butcher method, is defined as in Table II.
[1] V. I. Oseledec, Trans. Moscow Math. Soc. 19, 197 (1968).

[2] G. Benettin, L. Galgani, and J.-M. Strelcyn, Phys. Rev. A 14, 2338 (1976).

[3] M.-C. Firpo, Phys. Rev. E 57, 6599 (1998).

[4] P. Butera and G. Caravati, Phys. Rev. A 36, 962 (1987).

[5] G. Mutschke and U. Bahr, Physica D 69, 302 (1993).

[6] T. Konishi and K. Kaneko, J. Phys. A 25, 6283 (1992).

[7] S. Sasa, nlin.CD/0006042.

[8] M. Pettini, Phys. Rev. E 47, 828 (1993).

[9] L. Casetti, R. Livi, and M. Pettini, Phys. Rev. Lett. 74, 375 (1995).

[10] L. Casetti, C. Clementi, and M. Pettini, Phys. Rev. E 54, 5969 (1996).

[11] L. Casetti, M. Pettini, and E. G. D. Cohen, Phys. Rep. 337, 237 (2000).

[12] E. T. Whittaker, Analytical Dynamics of Particles and Rigid
Bodies (Cambridge University Press, Cambridge, England, 1937).

[13] C. P. Ong, Adv. Math. 15, 269 (1975).

[14] K. Yano and S. Ishihara, Tangent and Cotangent Bundles (Marcel Dekker, New York, 1973).

[15] S. Sasaki, Tohoku Math. J. 10, 338 (1958).

[16] H. Yoshida, Phys. Lett. A 150, 262 (1990).

[17] L. Casetti, Phys. Scr. 51, 29 (1995).

[18] E. Hairer, S. P. Nørsett, and G. Wanner, Solving Ordinary Differential Equations I, 2nd ed. (Springer-Verlag, Berlin, 1993).

[19] M. Cerruti-Sola, R. Franzosi, and M. Pettini, Phys. Rev. E 56, 4872 (1997).

[20] R. Livi, A. Politi, S. Ruffo, and A. Vulpiani, J. Stat. Phys. 46, 147 (1987).

[21] Y. Y. Yamaguchi, J. Phys. A 31, 195 (1998).

[22] Y. Y. Yamaguchi, Prog. Theor. Phys. 95, 717 (1996). 